\title{
CONVERGENCE TO EQUILIBRIUM FOR INTERMITTENT SYMPLECTIC MAPS
}

\author{
CARLANGELO LIVERANI, MARCO MARTENS
}

\begin{abstract}
We investigate a class of area preserving non-uniformly hyperbolic maps of the two torus. First we establish some results on the regularity of the invariant foliations, then we use this knowledge to estimate the rate of mixing.
\end{abstract}

\section{INTRODUCTION}

In recent years the physics community has devoted an increasing attention to anomalous properties of physical systems (e.g., anomalous transport, anomalous diffusion, anomalous conductivity, etc.). Such properties have proven relevant in many fields such as thermal conductivity, kinetic equations, plasma physics, etc. and they are widely believed to be dynamical in nature. In fact, such phenomena seem to depend on the weak chaotic properties of the underling dynamics, see [24] and references therein for a detailed discussion. The basic idea is that, while uniformly hyperbolic dynamics gives rise to normal transport properties (consider for example the diffusive behavior in a finite horizon Lorentz gas [4]) non-uniform hyperbolicity gives rise to different behavior (e.g. the anomalous diffusion believed to occur in infinite horizon Lorentz gas [3]) due to weaker mixing properties (e.g. polynomial decay of correlations) and regions in which the motions is rather regular and where the systems spend a substantial fraction of time (sticky regions).

Unfortunately, the theoretical understanding of dynamical models with polynomial decay of correlations is extremely limited, hence the necessity to rigorously investigate relevant toy models. The only well understood cases are expanding one dimensional maps with a neutral fixed point. Such maps were proposed as a model of intermittent behavior in fluids ([19]) and have been widely studied. It has been proven that such maps enjoy polynomial decay of correlations with the rate depending on the behavior of the fixed point [13, 22, 8, 9, 20, 5]. In addition, when the decay of correlation is sufficiently slow, the observables do not satisfy the Central Limit Theorem or the Invariance Principle but rather, when properly rescaled, some stable law (25, 6, 17]).

Some, more partial, results exist for multidimensional expanding maps [18] as well. Yet, the usual physically relevant models are connected to Hamiltonian dynamics and, to our knowledge, no rigorous results are available in such a situation. The simplest case which retain some Hamiltonian flavor is clearly a two dimensional area preserving map. In fact, mixing area preserving maps of the two dimensional torus with a neutral fixed point (the simplest type of sticky set) have been investigated numerically [1] predicting the possibility of a polynomial decay of correlations. In this paper we consider a class of non-uniformly hyperbolic symplectic maps (2.1) of the two torus where the hyperbolicity breaks down because of a non-hyperbolic fixed point. In fact, the linearized dynamics at the fixed point is a shear (2.3). We prove that the decay of correlations is polynomial and, more precisely, decays at least as $n^{-2}$ and, in some sense, one cannot expect much more. Note that the example treated numerically in [1] is a special case of the present setting. In [1] the predicted decay was $n^{-2.5}$. This emphasizes the difficulty to investigate such issues and the strong need for more theoretical work on the subject.

The result in the present paper is based on a precise quantitative analysis of the angle between the stable and the unstable direction. This angle turns out to degenerate approaching the origin (where the non hyperbolic fixed point is located). Once such a control is achieved it is possible to obtain a bound on the expansion and contraction in the system. Such expansion turns out to be only polynomial, in contrast with the uniformly hyperbolic case where it is exponential. In turn, the bound on the expansion allows

Date: November 13, 2018.

One of us (C.L.) would like to thank S.Vaienti and M.Benedicks for helpful discussions. In addition, we acknowledge the support from the ESF Program PRODYN, I.B.M., M.I.U.R. and NSF. 
to study the regularity of the stable and unstable foliation. It turns out that they are $\mathcal{C}^{1}$ away from the origin. This suffice to apply a simple random approximation technique that allows estimating the speed of the correlations.

As the rate of convergence to equilibrium is of order $n^{-2}$, see Theorem 2.4 the Central Limit Theorem holds for zero average observable, see Corollary 2.6 so the model does not exhibit anomalous statistical behavior in this respect. Yet, it clearly exhibits an intermittent behavior and it shows the mechanism whereby slow decay of correlations may arise. The present work emphasizes the need to carry out similar studies in cases where the set producing intermittency has a more complex structure than a simple isolated point.

The paper is organized as follows: section 2 details the model and makes precise the results. Section [3 studies the local dynamics at the fixed point and, in particular the properties of its stable and unstable manifolds. This can be achieved in many way, here we find most efficient to apply a variational technique. Section 4 establishes a precise bound for the angle between the stable and the unstable direction at each point. As anticipated, such a bound yields an a priory bound on the expansion and contractions rates in the systems, these are obtained in section 5 The latter result suffices to apply standard distortion estimates that, in turn, allow to prove precise results on the regularity of the invariant foliation and the holonomies, see section [6] and section 7 respectively. Next, in section 8 we introduce a random perturbation of the above map and investigate its statistical properties that, thanks to the added randomness, can be addressed fairly easily. The relevance of the above random perturbation is that the limit of zero noise allows to easily obtain a bound on the rate of mixing in the original map, we do this in section 9 Finally, in section [10] we show that the obtained bound is close to being optimal. The paper ends with Remark 10.2 pointing to the unsatisfactory nature of some of the present results and the need to investigate the related open problems.

\section{THE MODEL AND THE RESUlts}

For each $h \in \mathcal{C}^{\infty}\left(\mathbb{T}^{1}, \mathbb{T}^{1}\right)$ we define the map $T: \mathbb{T}^{2} \rightarrow \mathbb{T}^{2}$ by ${ }^{1}$

$$
T(x, y)= \begin{cases}x+h(x)+y & \bmod 1 \\ h(x)+y & \bmod 1\end{cases}
$$

We moreover require the following properties

(1) $h(0)=0$ (zero is a fixed point);

(2) $h^{\prime}(0)=0$ (zero is a neutral fixed point)

(3) $h^{\prime}(x)>0$ for each $x \neq 0$ (hyperbolicity)

Note that conditions (2-3) imply that zero is a minimum for $h^{\prime}$, which forces

$$
h^{\prime \prime}(0)=0 ; \quad h^{\prime \prime \prime}(0) \geq 0 .
$$

We will restrict to the generic case

(4) $h^{\prime \prime \prime}(0)>0$.

In order to simplify the discussion we will also assume the following symmetry

(5) $h(-x)=-h(x)$.

This means that we can write

$$
h(x)=b x^{3}+\mathcal{O}\left(x^{5}\right) .
$$

Remark 2.1. Note that two facts implied by the above assumptions are not necessary and could be done away with at the price of more extra work: the hypothesis that there is only one neutral fixed point (finitely many neutral periodic orbits would make little difference) and the symmetry (5). We assume such facts only to simplify the presentation of the arguments.

${ }^{1}$ Note that the following formula is equivalent, by the symplectic change of variable $q=x-y, p=y$, to the map

$$
\widetilde{T}(q, p)= \begin{cases}q+p & \bmod 1 \\ p+h(q+p) & \bmod 1\end{cases}
$$

which belongs to the standard map family. Yet, the functions $h$ considered here differ substantially from the sine function which would correspond to the classical Chirikov-Taylor well known example. 
Since the derivative of the map is given by

$$
D T=\left(\begin{array}{cc}
1+h^{\prime}(x) & 1 \\
h^{\prime}(x) & 1
\end{array}\right)
$$

$\operatorname{det}(D T)=1$, thus the Lebesgue measure $m$ is an invariant measure (the maps are symplectic). From now on we will consider the dynamical systems $T:\left(\mathbb{T}^{2}, m\right) \rightarrow\left(\mathbb{T}^{2}, m\right)$.

Formula (2.3) and property (3) imply that the cone $\mathcal{C}_{+}=\left\{v \in \mathbb{R}^{2} \mid Q(v):=\left\langle v_{1}, v_{2}\right\rangle \geq 0\right\}$ is invariant for $D T$. In additions, it is easy to check that $D_{\xi} T^{2} \mathcal{C}_{+} \subset \operatorname{int} \mathcal{C}_{+} \cup\{0\}$ for all $\xi \in \mathbb{T}^{2} \backslash\{0\}$. From this and the general theory, see [14], follows immediately

Theorem 2.2. The above described dynamical systems are non-uniformly hyperbolic and mixing.

Example 1. An interesting concrete example for the above setting is given by the function $h(x):=x-\sin x$.

The question remains about the rate of mixing, this is the present topic.

Remark 2.3. In the following by $\mathrm{C}$ we designate a generic constant depending only on $T$. Accordingly, its value may vary from an occurrence to the next. In the instances when we will need a constant of the above type but with a fixed value we will use sub-superscripts.

Theorem 2.4. For each $f, g \in \mathcal{C}^{1}\left(\mathbb{T}^{2}, \mathbb{R}\right), \int f=0$, holds true $e^{2}$

$$
\left|\int f g \circ T^{n}\right| \leq \mathrm{C}\|f\|_{\mathcal{C}^{1}}\|g\|_{\mathcal{C}^{1} n^{-2}}(\ln n)^{4} .
$$

Remark 2.5. As in other similar cases [13, 10, 16] the logarithmic correction is almost certainly an artifact of the technique of the proof. It could probably be removed by using a more sophisticated (and thus more technically involved) approach. See also section 10.

Form Theorem 2.4 many facts follow, just to give an example let us mention the following result that can be obtained from Theorem 1.2 in [12].

Corollary 2.6 (CLT). Given $f \in \mathcal{C}^{1}, \int f=0$, the random variable

$$
\frac{1}{\sqrt{n}} \sum_{i=0}^{n-1} f \circ T^{i}
$$

converges in distribution to a Gaussian variable with zero mean and finite variance $\sigma$. In addition, $\sigma=0$ iff there exists $\varphi \in L^{1}$ such that $f=\varphi-\varphi \circ T .^{3}$

The rest of the paper is devoted to the proof of Theorem 2.4 that will find its conclusion in section 9 The basic fact needed in the proof, a fact of independent interest and made quantitatively precise in Lemma 6.3. is the following.

Theorem 2.7. The stable and unstable distributions are $\mathcal{C}^{1}$ in $\mathbb{T}^{2} \backslash\{0\}$.

\section{THE FIXED POINT MANIFOLDS}

As usual we start by studying the local dynamics near the fixed point. The first basic fact is the existence of stable and unstable manifolds. This is rather standard, yet since we need some quantitative information we will construct them explicitly.

Instead of constructing them via usual fixed point arguments it turns out to be faster to use a variational method.

\footnotetext{
${ }^{2}$ In fact, a slightly sharper bound holds, see 9.1 .

${ }^{3}$ In particular this means that the average of $f$ on each periodic orbit must be zero.
} 
3.1. A variational argument. Let us consider, in a neighborhood of zero, the function

$$
L\left(x, x_{1}\right):=\frac{1}{2}\left(x-x_{1}\right)^{2}+G(x) ; \quad G(x):=\int_{0}^{x} h(z) d z .
$$

By setting

$$
\begin{aligned}
& y:=-\frac{\partial L}{\partial x}=x_{1}-x-h(x) \\
& y_{1}:=\frac{\partial L}{\partial x_{1}}=x_{1}-x
\end{aligned}
$$

we have $\left(x_{1}, y_{1}\right)=T(x, y)$, that is $L$ is a generating function for the map (2.1).

Then, for each $a \in \mathbb{R}$, we define the Lagrangian $\mathcal{L}_{a}: \ell^{2}(\mathbb{N}) \rightarrow \mathbb{R}$ by

$$
\mathcal{L}_{a}(x):=\sum_{n=1}^{\infty} L\left(x_{n}, x_{n+1}\right)+L\left(a, x_{1}\right)
$$

The justification of the above definition rests in the following Lemma.

Lemma 3.1. For each $a \in(-1,1)$, holds true $\mathcal{L}_{a} \in \mathcal{C}^{1}\left(\ell^{2}(\mathbb{N})\right)$. In addition, if $x \in \ell^{2}(\mathbb{N})$ is such that $D_{x} \mathcal{L}_{a}=0$, then setting $x_{0}=a$ and $y_{n}:=x_{n+1}-x_{n}-h\left(x_{n}\right)$, we have $T^{n}\left(x_{0}, y_{0}\right)=\left(x_{n}, y_{n}\right)$.

Proof. First of all (2.2) implies that there exists $\mathrm{C}>0$ such that $|G(x)| \leq \mathrm{C} x^{4}$. It is then easy to see that $\mathcal{L}_{a}$ is well defined for each sequence in $\ell^{2}(\mathbb{N})$.

Next, for each $n \in \mathbb{N}$ let us define $\left(\nabla \mathcal{L}_{a}\right)_{n}:=\partial_{x_{n}} \mathcal{L}_{a}$. Clearly,

$$
\begin{aligned}
& \left(\nabla \mathcal{L}_{a}\right)_{1}(x)=2 x_{1}-x_{2}+h\left(x_{1}\right)-a \\
& \left(\nabla \mathcal{L}_{a}\right)_{n}(x)=2 x_{n}-x_{n+1}-x_{n-1}+h\left(x_{n}\right) .
\end{aligned}
$$

Of course, for $x \in \ell^{2}(\mathbb{N}), \nabla \mathcal{L}_{a}(x) \in \ell^{2}(\mathbb{N})$, it is then trivial to check that $D_{x} \mathcal{L}_{a}(v)=\left\langle\nabla \mathcal{L}_{a}(x), v\right\rangle$. The last statement follows by a direct computation.

By the above Lemma it is clear that one can obtain the stable manifolds of the fixed point from the critical points of $\mathcal{L}_{a}$, it remains to prove that such critical points do exist. We will start by considering the case $a \geq 0$.

Define

$$
Q_{B}:=\left\{x \in \ell^{2}(\mathbb{N})|| x_{n}-\frac{A}{n+c} \mid \leq B(n+c)^{-\frac{3}{2}}\right\}
$$

where $A:=\sqrt{\frac{2}{b}} ; c:=\frac{A}{a}$.

It is immediate to check that $Q_{B}$ is compact and convex. In addition, if $a$ is sufficiently small, then $G$ is strictly convex on $[-2 a, 2 a]$ which implies that $\left.\mathcal{L}_{a}\right|_{Q_{B}}$ is strictly convex. Accordingly, $\mathcal{L}_{a}$ has minimum in $Q_{B}$, moreover the strict convexity implies that such a minimum is unique, for $a$ fixed.

Let us call $x(a)$ the point in $Q_{B}$ where $\mathcal{L}_{a}$ attains its minimum.

Lemma 3.2. For a small enough, $D_{x(a)} \mathcal{L}_{a}=0$.

Proof. Suppose that $\partial_{x_{n}} \mathcal{L}_{a}(x(a)) \neq 0$ for some $n \in \mathbb{N}$, for example suppose it is negative. Then $x(a)$ is on the border of $Q_{B}$, say $x(a)_{n}=A(n+c)^{-1}+B(n+c)^{-\frac{3}{2}}$, otherwise we could increase $x(a)_{n}$ and decrease $\mathcal{L}_{a}$ 
still remaining in $Q_{B}$, contrary to the assumption. But then

$$
\begin{aligned}
\partial_{x_{n}} \mathcal{L}_{a}(x(a)) & =2 x(a)_{n}-x(a)_{n-1}-x(a)_{n+1}+h\left(x(a)_{n}\right) \\
& =\frac{2 A}{n+c}+\frac{2 B}{(n+c)^{\frac{3}{2}}}-x(a)_{n-1}-x(a)_{n+1}+h\left(\frac{A}{n+c}+\frac{B}{(n+c)^{\frac{3}{2}}}\right) \\
& \geq-\frac{2 A}{\left[(n+c)^{2}-1\right](n+c)}+\frac{2 A}{(n+c)^{3}}+\frac{3 b A^{2} B}{(n+c)^{\frac{7}{2}}}+\mathcal{O}\left((n+c)^{-4}\right) \\
& +\frac{B(n+c)^{3}\left\{2\left(1-(n+c)^{-2}\right)^{\frac{3}{2}}-\left(1-(n+c)^{-1}\right)^{\frac{3}{2}}-\left(1+(n+c)^{-1}\right)^{\frac{3}{2}}\right.}{(n+c)^{\frac{3}{2}}\left[(n+c)^{2}-1\right]^{\frac{3}{2}}} \\
& =\frac{\left(6-\frac{15}{4}\right) B}{(n+c)^{\frac{7}{2}}}+\mathcal{O}\left((n+c)^{-4}\right) \geq 0
\end{aligned}
$$

provided $a$ is sufficiently small. We have thus a contradiction. The other possibilities are analyzed similarly.

To conclude we need some information on the regularity of $x(a)$ as a function of $a$. Unfortunately, the implicit function theorem does not applies since $D^{2} \mathcal{L}_{a}$ does not have a spectral gap, yet for our purposes a simple estimate suffices.

Lemma 3.3. $x_{1}(a)$ is a Lipschitz function of a. Moreover, when derivable,

$$
\left|y_{0}(a)^{\prime}\right| \leq \mathrm{C}\left|x(a)_{0}\right| \text {. }
$$

Proof. By Lemma 3.2 it follows, for each $a, a^{\prime}$ sufficiently small

$$
\partial_{x_{n}} \mathcal{L}_{a^{\prime}}\left(x\left(a^{\prime}\right)\right)=\partial_{x_{n}} \mathcal{L}_{a}(x(a))=0
$$

that is

$$
\partial_{x_{n}} \mathcal{L}_{a^{\prime}}\left(x\left(a^{\prime}\right)\right)-\partial_{x_{n}} \mathcal{L}_{a^{\prime}}(x(a))=\partial_{x_{n}} \mathcal{L}_{a}(x(a))-\partial_{x_{n}} \mathcal{L}_{a^{\prime}}(x(a))
$$

which yields

$$
\begin{aligned}
& \left(2+h^{\prime}\left(\xi_{1}\right)\right) \zeta_{1}-\zeta_{2}=a^{\prime}-a \\
& \left(2+h^{\prime}\left(\xi_{n}\right)\right) \zeta_{n}-\zeta_{n+1}-\zeta_{n-1}=0,
\end{aligned}
$$

where $\zeta_{n}:=x\left(a^{\prime}\right)_{n}-x(a)_{n}$ and $\xi_{n} \in\left[x(a)_{n}, x\left(a^{\prime}\right)_{n}\right]$.

Notice that, if $\left|\zeta_{n}\right| \geq\left|\zeta_{n-1}\right|$, then

$$
\left|\zeta_{n+1}\right|=\left|\left(2+h^{\prime}\left(\xi_{n}\right)\right) \zeta_{n}-\zeta_{n-1}\right| \geq 2\left|\zeta_{n}\right|-\left|\zeta_{n-1}\right| \geq\left|\zeta_{n}\right| .
$$

Thus, by induction, if $\left|\zeta_{n}\right| \geq\left|\zeta_{n-1}\right|$, then $\left|\zeta_{m}\right| \geq\left|\zeta_{n-1}\right|$ for each $m \geq n$, which would imply $\zeta_{n-1}=0$ since $\zeta \in \ell^{2}(\mathbb{N})$. But then $\left(2+h^{\prime}\left(\xi_{n}\right)\right) \zeta_{n}=\zeta_{n+1}$, that is $\left|\zeta_{n+1}\right| \geq\left|\zeta_{n}\right|$. Accordingly, again by induction, $\zeta_{m}=0$ for each $m \geq n-1$. This means that we can restrict ourselves to the case $\zeta_{n} \neq 0,\left|\zeta_{n}\right| \geq\left|\zeta_{n+1}\right|$. Hence,

$$
\left|a^{\prime}-a\right|=\left|\left(2+h^{\prime}\left(\xi_{1}\right)\right) \zeta_{1}-\zeta_{2}\right| \geq 2\left|\zeta_{1}\right|-\left|\zeta_{2}\right| \geq\left|\zeta_{1}\right| .
$$

That is $\left|x\left(a^{\prime}\right)_{n}-x(a)_{n}\right| \leq\left|x_{1}\left(a^{\prime}\right)-x_{1}(a)\right| \leq\left|a^{\prime}-a\right|$.

Finally, summing (3.4) over $n \in \mathbb{N}$,

$$
\sum_{n=1}^{\infty} h^{\prime}\left(\xi_{n}\right) \zeta_{n}=-\zeta_{1}+a^{\prime}-a .
$$

Thus, where all the $x(a)_{n}$ are differentiable (a full measure set), $\left|x(a)_{n}^{\prime}\right| \leq\left|x(a)_{1}^{\prime}\right| \leq 1$ and

$$
\begin{aligned}
& x(a)_{1}^{\prime}=1-\sum_{n=1}^{\infty} h^{\prime}\left(x(a)_{n}\right) x(a)_{n}^{\prime} \\
& y(a)_{0}^{\prime}=-\sum_{n=0}^{\infty} h^{\prime}\left(x_{n}\right) x(a)_{n}^{\prime} .
\end{aligned}
$$


Accordingly,

$$
\left|y_{0}(a)^{\prime}\right| \leq 6 b \sum_{n=0}^{\infty} x_{n}^{2} \leq \mathrm{C} a
$$

Clearly, the above Lemma implies that, calling $\left(x, \gamma_{s}(x)\right)$ the graph of the stable manifold, $\gamma_{s} \in \operatorname{Lip}(-1,1)$. The case $a \leq 0$ and the unstable manifolds can be treated similarly, yet there exists a faster-and more instructive-way.

3.2. Reversibility. Notice that the map $T$ is reversible with respect to the transformations ${ }^{4}$

$$
\Pi(x, y):=(x,-y-h(x)) ; \quad \Pi_{1}(x, y):=(-x, y+h(x))
$$

Indeed, $\Pi^{2}=\Pi_{1}^{2}=\mathbf{I d}$ and $\Pi T \Pi=\Pi_{1} T \Pi_{1}=T^{-1}$.

Remark 3.4. The reversibility implies that, for $x \geq 0,\left(x, \gamma_{u}(x)\right)=\Pi\left(x, \gamma_{s}(x)\right)$, and, for $x \leq 0,\left(x, \gamma_{u}(x)\right)=$ $\Pi_{1}\left(-x, \gamma_{s}(-x)\right)$ is the unstable manifold of zero.

3.3. A quasi-Hamiltonian. To study the motion near the fixed point it is helpful to find a local "Hamiltonian" function. By Hamiltonian function we mean a function that is locally invariant for the dynamics. Such a function can be computed as a formal power series starting by the relation $H \circ T=H$. In fact, we are interested only in a suitable approximation. A direct computation yields that, by defining $G(x):=\int_{0}^{x} h(z) d z$ and

holds true ${ }^{5}$

$$
H(x, y):=\frac{1}{2} y^{2}-G(x)+\frac{1}{2} h(x) y-\frac{1}{12} h^{\prime}(x) y^{2}+\frac{1}{12} h(x)^{2},
$$

This approximate conservation law suffices to obtain rather precise information on the near fixed point dynamics. ${ }^{6}$ The first application is given by the following information on the stable manifold.

Lemma 3.5. For $x \geq 0$ sufficiently small holds

$$
\gamma_{s}(x)=-A^{-1} x^{2}+\mathcal{O}\left(x^{3}\right) .
$$

Proof. Using the notation of Lemma 3.2 for fixed $a$ we get

$$
y_{n}=x_{n+1}-x_{n}-h\left(x_{n}\right)=\mathcal{O}\left((n+c)^{-\frac{3}{2}}\right) .
$$

Hence

Using equation (3.8) we have

$$
H\left(x_{n}, y_{n}\right)=\mathcal{O}\left((n+c)^{-3}\right) .
$$

$$
H\left(x_{0}, y_{0}\right)=H\left(x_{n}, y_{n}\right)+\mathcal{O}\left(\sum_{i=0}^{n-1} x_{i}^{8}+y_{i}^{4}\right)
$$

that is

$$
\left|H\left(x_{0}, y_{0}\right)\right| \leq \mathrm{C}\left\{(n+c)^{-3}+\sum_{i=0}^{\infty}(n+c)^{-6}\right\} \leq \mathrm{C}\left\{(n+c)^{-3}+c^{-5}\right\} .
$$

Taking the limit for $n$ to infinity in the above expression and remembering the definition of $c$ follows

$$
\left|H\left(x_{0}, y_{0}\right)\right| \leq \mathrm{C} x_{0}^{5} .
$$

\footnotetext{
${ }^{4}$ While the reversibility for $\Pi$ is a general fact, the one for $\Pi_{1}$ depends on the simplifying symmetry hypothesis (5).

${ }^{5}$ In fact, setting $\left(x_{1}, y_{1}\right):=T(x, y)$, holds

$$
\begin{aligned}
& H\left(x_{1}, y_{1}\right)-H(x, y)=y h(x)+\frac{1}{2} h(x)^{2}-h(x) y-h(x)^{2}-\frac{1}{2} h^{\prime}(x) y_{1}^{2}-\frac{1}{6} h^{\prime \prime}(x) y_{1}^{3}+\frac{1}{2} h^{\prime}(x) y_{1}^{2} \\
& +\frac{1}{2} h(x)^{2}+\frac{1}{4} h^{\prime \prime}(x) y_{1}^{3}-\frac{1}{12} h^{\prime \prime}(x) y_{1}^{3}-\frac{1}{6} h^{\prime}(x) h(x) y_{1}+\frac{1}{6} h^{\prime}(x) h(x) y_{1}+\mathcal{O}\left(x^{8}+x^{4} y^{2}+y^{4}\right) .
\end{aligned}
$$

${ }^{6}$ The reader should be aware that it is possible to do much better, that is to obtain an exponentially precise conservation law, see [1], 2].
} 
Since equation (3.9) implies $\left|y_{0}\right|=\mathcal{O}\left(x_{0}^{\frac{3}{2}}\right)$, from (3.7) we have

$$
y_{0}^{2}-\frac{b}{2} x_{0}^{4}+b x_{0}^{3} y_{0}=\mathcal{O}\left(x_{0}^{5}\right)
$$

from which the lemma follows.

According to Lemma 3.5 the local picture of the manifolds is given by Figure 1

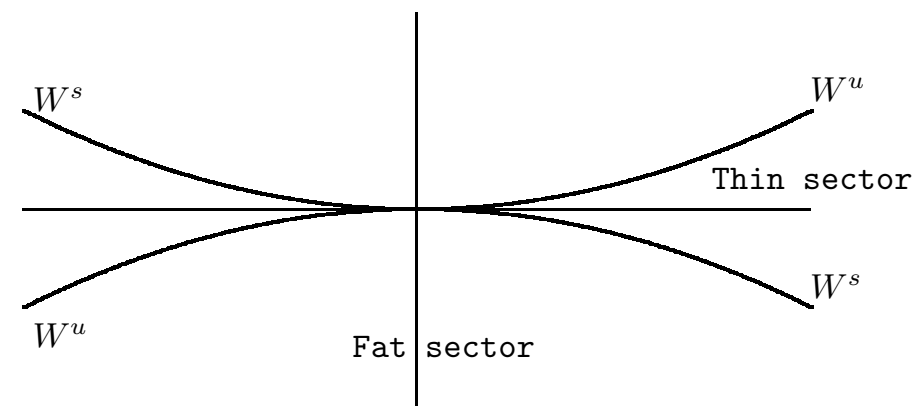

FIgURE 1. The manifolds of the fixed point

3.4. Manifold regularity. Since in the previous section we have seen that the manifold are Lipschitz curves, we can define the dynamics restricted to the unstable manifold:

$$
f_{u}(x):=x+h(x)+\gamma_{u}(x) .
$$

Our next task is to obtain sharper information on the manifolds regularity. ${ }^{7}$

Lemma 3.6. The unstable manifold of the fixed point is $\mathcal{C}^{2}$, apart from zero.

Proof. It is clearly enough to show that $\gamma_{u} \in \mathcal{C}^{2}$ apart from zero. To do so call $u(x)=\gamma_{u}^{\prime}(x)$ (the derivative exists almost everywhere since $\gamma_{u}$ is Lipschitz). The tangent vector to the unstable manifold has the form $(1, u)$. On the other hand

$$
\begin{aligned}
\lambda_{u}\left(f_{u}^{-1}(x)\right)\left(\begin{array}{c}
1 \\
u(x)
\end{array}\right) & :=\left(\begin{array}{cc}
1+h^{\prime}\left(f_{u}^{-1}(x)\right) & 1 \\
h^{\prime}\left(f_{u}^{-1}(x)\right) & 1
\end{array}\right)\left(\begin{array}{c}
1 \\
u\left(f_{u}^{-1}(x)\right)
\end{array}\right) \\
& =: \lambda_{u}\left(f_{u}^{-1}(x)\right)\left(\begin{array}{c}
1 \\
F\left(f_{u}^{-1}(x), u\left(f_{u}^{-1}(x)\right)\right)
\end{array}\right)
\end{aligned}
$$

where

$$
\begin{aligned}
& \lambda_{u}(x):=1+h^{\prime}(x)+u(x)=f_{u}^{\prime}(x) \\
& F(x, u):=1-\frac{1}{1+h^{\prime}(x)+u} .
\end{aligned}
$$

Accordingly, setting $x_{i}:=f_{u}^{-i}(x)$, holds $u\left(x_{i}\right)=F\left(x_{i+1}, u\left(x_{i+1}\right)\right)$. Next, let $v_{i}:=2 A^{-1} x_{i}$, then a direct computation yields $F\left(x_{i}, v_{i}\right)-v_{i-1}=\mathcal{O}\left(x_{i}^{3}\right)$, thus

$$
\left|u\left(x_{i}\right)-v_{i}\right| \leq\left|F\left(x_{i+1}, u\left(x_{i+1}\right)\right)-F\left(x_{i+1}, v_{i+1}\right)\right|+\mathrm{C} x_{i}^{3} \leq\left|u\left(x_{i+1}\right)-v_{i+1}\right|+\mathrm{C} x_{i}^{3} .
$$

By induction, and equation (3.3), it follows $\left|u(x)-2 A^{-1} x\right| \leq \mathrm{C} \sum_{i=0}^{\infty} x_{i}^{3} \leq \mathrm{C} x^{2}$.

On the other hand, given a different point $z$, it holds

$$
\begin{aligned}
u(x)-u(z) & =F\left(x_{1}, u\left(x_{1}\right)\right)-F\left(z_{1}, u\left(z_{1}\right)\right) \\
& \left.=\lambda_{u}\left(x_{1}\right)^{-1} \lambda_{u}\left(z_{1}\right)^{-1}\left\{\left(u\left(x_{1}\right)-u\left(z_{1}\right)\right)+h^{\prime}\left(x_{1}\right)-h^{\prime}\left(z_{1}\right)\right)\right\} .
\end{aligned}
$$

${ }^{7}$ Of course, the manifold should be as smooth as $h$, but this results is not needed in the following while we do need an explicit bound on the curvature. 
Iterating the above equation yields

$$
\begin{aligned}
u(x)-u(z)= & \lambda_{u, n}(x)^{-1} \lambda_{u, n}(z)^{-1}\left(u\left(x_{n}\right)-u\left(z_{n}\right)\right) \\
& +\sum_{k=1}^{n} \lambda_{u, k}(x)^{-1} \lambda_{u, k}(z)^{-1}\left(h^{\prime}\left(x_{k}\right)-h^{\prime}\left(z_{k}\right)\right)
\end{aligned}
$$

where $\lambda_{u, n}(x):=\prod_{k=1}^{n} \lambda_{u}\left(f_{u}^{-k}(x)\right)$. Next, let $x=a$, then, accordingly to Lemma 3.2, equation (3.3) and Remark 3.4 we have $x_{i}=A(i+c)^{-1}+\mathcal{O}\left((i+c)^{3 / 2}\right)$. This means that, in a sufficiently small neighborhood of zero, and for $z$ sufficiently close to $x$ holds

$$
\begin{aligned}
\lambda_{u, n}(x) & \geq \prod_{k=1}^{n}\left(1+v_{k}-\mathrm{C} x_{i}^{2}\right) \geq \prod_{k=1}^{n}\left(1+\frac{2}{k+c}-\mathrm{C}(k+c)^{-3 / 2}\right) \\
& \geq e^{\sum_{k=1}^{n} \frac{2}{k+c}-\mathrm{C}(k+c)^{-3 / 2}} \geq \mathrm{C}\left(A^{-1} a n+1\right)^{2}
\end{aligned}
$$

provided $x$ is close enough to zero. The same estimate holds for $\lambda_{u, n}(z)$.

This implies that $u$ is continuous. Indeed, for each $\varepsilon>0$ choose $n_{0}(x) \in \mathbb{N}$ such that $\lambda_{u, n_{0}(x)}\left(x_{n}\right)^{-1} \leq \varepsilon$, then

$$
|u(x)-u(z)| \leq \sum_{k=1}^{n_{0}(x)}\left|h^{\prime}\left(x_{k}\right)-h^{\prime}\left(z_{k}\right)\right|+\varepsilon
$$

and we can thus choose $z$ close enough to $x$ such that $|u(x)-u(z)| \leq 2 \varepsilon$. Note that this implies the continuity of the $\lambda_{u, n}$ as well.

To conclude, we choose $n(z)$ such that $\mathrm{C}\left(A^{-1} \operatorname{an}(z)+1\right)^{-4} \geq|x-z|^{1+\alpha}$, for some $\alpha>0$, accordingly

$$
u(x)-u(z)=\sum_{k=1}^{n(z)} \lambda_{u, k}(x)^{-1} \lambda_{u, k}(z)^{-1}\left(h^{\prime}\left(x_{k}\right)-h^{\prime}\left(z_{k}\right)\right)+\mathcal{O}\left(|x-z|^{1+\alpha}\right)
$$

Since the series is uniformly convergent we have

$$
u^{\prime}(x)=\sum_{k=1}^{\infty} \lambda_{u, k}(x)^{-3} h^{\prime \prime}\left(x_{k}\right)
$$

from which the lemma follows. ${ }^{8}$

Remark 3.7. All the above results for the unstable manifold $\gamma_{u}$ have the obvious counterpart for the stable manifold $\gamma_{s}$ that can be readily obtained via reversibility, see Remark 3.4.

\section{A NARROWER CONE FIELD}

Here our goal is to estimate the angle between stable and unstable manifolds.

More precisely, we wish to prove that there exists two constants $K_{+}, K_{-} \in \mathbb{R}^{+}$such that the cone field $\mathcal{C}_{*}(\xi):=\left\{(1, u) \in \mathbb{R}^{2} \mid K_{-}(|x|+\sqrt{|y|}) \leq u \leq K_{+}(|x|+\sqrt{|y|})\right\}$ contains the unstable direction (by reversibility we can also define the stable cone field $\left.\mathcal{C}_{*}^{-}\right)$.

Proposition 4.1. For each $\xi \in \mathbb{T}^{2}$ holds true $E^{u}(\xi) \in \mathcal{C}_{*}(\xi), E^{s}(\xi) \in \mathcal{C}_{*}^{-}(\xi)$.

The rest of the section is devoted to the proof of Proposition 4.1 .

Clearly a problem arises only in a neighborhood of zero. Accordingly the first step is to gain a better understanding of the dynamics near zero.

\footnotetext{
${ }^{8}$ Remark that to obtain the result on a larger neighborhood it suffices to iterate the unstable manifold forward.
} 
4.1. Near fixed point dynamics. For each $\delta>0$ let $Q_{\delta}:=[-\delta, \delta]^{2}$ be a square neighborhood of zero. The manifolds of the fixed point divide $Q_{\delta}$ into four sectors: two thin and two fat (see Figure 1). We will discuss explicitly the dynamics in the two sectors below the unstable manifold (the other two being identical by symmetry).

Lemma 4.2. For each $(x, y) \in \mathbb{T}^{2}, y \leq \gamma_{u}(x)$, let $\left(x_{n}, y_{n}\right):=T^{n}(x, y)$, then it holds true

$$
x_{n} \leq f_{u}^{n}(x) \quad \forall n \geq 0 .
$$

Proof. First note that the trajectory will always remain below the unstable manifold. Hence, by induction,

$$
\begin{aligned}
x_{n+1} & =x_{n}+h\left(x_{n}\right)+y_{n} \leq x_{n}+h\left(x_{n}\right)+\gamma_{u}\left(x_{n}\right) \\
& \leq f_{u}^{n}(x)+h\left(f_{u}^{n}(x)\right)+\gamma_{u}\left(f_{u}^{n}(x)\right)=f_{u}^{n+1}(x) .
\end{aligned}
$$

The above lemma will suffice to control the dynamics in the thin sector, more work is needed for the fat one. In fact, when the trajectories are close to the stable or the unstable manifolds the above result can still be used (possibly remembering reversibility). On the other hand when the trajectory is close enough to zero its behavior is drastically different from the one on the invariant manifolds.

To define more precisely the meaning of "close to zero" let us introduce the parabolic sector $P_{M}:=$ $\left\{(x, y) \in Q_{1}|| y \mid \geq M x^{2}\right\}$. We consider a backward trajectory starting from $x \leq 0, y \leq \gamma_{u}(x)$, the other possibilities follow by reversibility. Let, as usual, $\left(x_{n}, y_{n}\right):=T^{n}(x, y), n \in \mathbb{Z}$. Let $m_{+}$be the smallest integer for which $\left(x_{-n}, y_{-n}\right) \in P_{M}, m$ the largest integer such that $x_{-m} \leq 0$, and $m_{-}$the largest integer for which $\left(x_{-n}, y_{-n}\right) \in P_{M}$. Define, see (3.7),

$$
E:=H\left(x_{-m}, y_{-m}\right) .
$$

In addition, define the function $\Upsilon_{E}:[-1,1] \rightarrow \mathbb{R}^{-}$, by ${ }^{9}$

$$
H\left(x, \Upsilon_{E}(x)\right)=E .
$$

Then, by (3.8), and since $\left|y_{-n}\right| \geq \mathrm{C} x_{-n}^{2}$,

$$
\begin{gathered}
H\left(x_{-n}, y_{-n}\right)-H\left(x_{-n}, \Upsilon_{E}\left(x_{-n}\right)\right)=H\left(x_{-n}, y_{-n}\right)-H\left(x_{-m}, y_{-m}\right) \leq \mathrm{C} \sum_{k=n}^{m} y_{-k}^{4} \\
\leq \mathrm{C} \sum_{k=n}^{m}\left|y_{-k}^{3}\right|\left(x_{-k-1}-x_{-k}\right) \leq \mathrm{C}\left|y_{-n}^{3}\right|\left|x_{-n}\right| .
\end{gathered}
$$

Accordingly, for $n \leq m$ it holds true

$$
\left|y_{-n}-\Upsilon_{E}\left(x_{-n}\right)\right| \leq \mathrm{C}\left|y_{-n}\right|^{2}\left|x_{-n}\right| .
$$

Lemma 4.3. In the above described situation, setting $M=\sqrt{b}$, the following holds true

(1) $f_{u}^{-n}(x) \leq x_{-n} \quad \forall n \leq m$;

(2) $f_{s}^{k}\left(x_{-n}\right) \geq x_{-n+k} \quad \forall n \geq m_{-}, k \leq n-m_{-}$;

(3) $\sqrt{E} \leq\left|y_{-n}\right| \leq 3 \sqrt{E}$ for all $n \in\left\{m_{+}, \ldots, m_{-}\right\}$;

(4) $m_{+} \leq 2 A\left(E b^{-1}\right)^{-\frac{1}{4}}$;

(5) $2(12 E b)^{-\frac{1}{4}} \leq m_{-}-m_{+} \leq 4(E b)^{-\frac{1}{4}}$.

Proof. The first fact is proven as in Lemma 4.2 the second follows by reversibility. Hence, by the results of section 3 for $n \leq m$, it follows

$$
\left|x_{-n}\right| \leq\left|f_{u}^{-n}(x)\right| \leq \frac{2 A|x|}{|x| n+A} .
$$

${ }^{9}$ Computing for $y \leq 0$ yields

$$
\begin{aligned}
\Upsilon_{E}(x) & =-\frac{h(x)}{2}-\sqrt{\frac{h(x)^{2}}{4}+2\left[G(x)+E-\frac{1}{12} h(x)^{2}\right]\left(1-\frac{1}{6} h^{\prime}(x)\right)} \\
& =-\sqrt{2(E+G(x))}\left(1+\mathcal{O}\left(x^{2}\right)\right)+\mathcal{O}\left(x^{3}\right) .
\end{aligned}
$$


Next we want to determine the points $x_{m_{+}}$and $x_{m_{-}}$. The idea is to use (4.2) that determines with good precision the geometry of the trajectories. Let $\bar{x}$ be defined by $\Upsilon_{E}(\bar{x})=-M \bar{x}^{2}$. Then

$$
\bar{x}=-\left[\frac{2 E}{M^{2}-\frac{b}{2}}\right]^{\frac{1}{4}}+\mathcal{O}\left(E^{\frac{5}{4}}\right) .
$$

On the other hand, since by definition $\left|y_{-m_{+}}\right| \geq M x_{-m_{+}}^{2}$ and $\left|y_{-m_{+}+1}\right| \leq M x_{-m_{+}+1}^{2}$, holds $\mid y_{-m_{+}-}$ $M x_{-m_{+}}^{2}|\leq \mathrm{C}| x_{-m_{+}}^{3} \mid$. Hence, by (4.2) it follows

$$
\left|x_{-m_{+}}-\bar{x}\right| \leq \mathrm{C} \frac{x_{-m_{+}}^{3}}{\min \left\{\bar{x}, x_{-m_{+}}\right\}} \leq \mathrm{C} \frac{x_{-m_{+}}^{3}}{x_{-m_{+}}-\left|x_{-m_{+}}-\bar{x}\right|} .
$$

Solving the above inequality yields

$$
\left|x_{-m_{+}}-\bar{x}\right| \leq \mathrm{C} x_{-m_{+}}^{2} \leq \mathrm{C} \bar{x}^{2} \leq \mathrm{C} \sqrt{E} .
$$

Analogously $\left|x_{-m_{-}}+\bar{x}\right| \leq \mathrm{C} \sqrt{E}$. From this (3) and (4) easily follows. Finally,

$$
2|\bar{x}| \geq\left|x_{m_{-}}-x_{m_{+}}\right|=\left|\sum_{n=m_{+}}^{m_{-}} y_{n}\right| \geq \mathrm{C}\left(m_{+}-m_{-}\right) \sqrt{E} .
$$

Which implies (5).

We are now ready to refine our knowledge of the stable and unstable direction. Let us fix $\varrho \in(0,1 / 2)$.

4.2. The cone field-Outside $Q_{\varrho}$. The general idea is to take the positive cone field $\mathcal{C}_{+}$(which is invariant and contains the unstable direction) and to push it forward in order to obtain a narrower cone field. First of all outside $Q_{\sqrt{\varrho}}$ we have (see (3.11)

$$
1 \geq F(x, u) \geq F(x, 0) \geq 2 b \varrho,
$$

where we have chosen $\varrho$ small enough. Hence the cone field $\mathcal{C}_{0}=\{(1, u) \mid 1 \geq u \geq 2 b \varrho\}$ is invariant outside $Q_{\sqrt{\varrho}}$. It remains to understand what happens in a neighborhood of the origin of order $\sqrt{\varrho}$.

Let us define $\bar{u}(\xi)$ by the equation $u=F(\xi, u)$. An easy computation shows

$$
\bar{u}(\xi)=\frac{-h^{\prime}(x)+\sqrt{h^{\prime}(x)^{2}+4 h^{\prime}(x)}}{2}=\sqrt{3 b}|x|+\mathcal{O}\left(x^{2}\right) .
$$

By reversibility we can restrict ourselves to the case $x \geq 0$, in this case the only possibility to enter the region $Q_{\sqrt{\varrho}}$ is via the fourth quadrant.

Note that $F(\xi, u) \geq u$ provided $0 \leq u \leq \bar{u}(\xi)$. This means that if $\xi \notin Q_{\sqrt{\varrho}}$ but $T \xi \in Q_{\sqrt{\varrho}}$, then the lower bound of the cone $D_{\xi} T^{n} \mathcal{C}_{0}$ does not decreases until $\sqrt{3 b} x_{i} \leq 2 b \varrho$, where $\left(x_{i}, y_{i}\right):=T^{i} \xi$.

Accordingly, the cone field $\mathcal{C}_{0}$ is invariant also in the fourth quadrant, outside the set $Q_{2 \sqrt{\frac{b}{3}} \varrho}$. Now consider the cone field $\mathcal{C}_{1}(\xi):=\{(1, u) \mid 2 b \varrho \leq u \leq L \bar{u}(\xi)\}, L=(3 b \varrho)^{-\frac{1}{2}}$, for $\xi \in Q_{\sqrt{\varrho}} \backslash Q_{2 \sqrt{\frac{b}{3}}}$. Clearly, if $|x| \geq \sqrt{\varrho}$, then $L \bar{u}(\xi) \geq 1$. Hence, as the point enters $Q_{\sqrt{\varrho}}$, the image of $\mathcal{C}_{0}$ is contained in $\mathcal{C}_{1}$, moreover we have already seen that the lower bound is invariant provided $\xi_{i} \notin Q_{2 \sqrt{\frac{b}{3}} \varrho}$. Let us follow the upper edge, if $u \leq L \bar{u}\left(\xi_{i}\right)$, then $^{10}$

$$
\begin{aligned}
& F\left(\xi_{i}, u\right)=F\left(\xi_{i}, u\right)-F\left(\xi_{i}, \bar{u}\left(\xi_{i}\right)\right)+\bar{u}\left(\xi_{i}\right) \\
& \quad \leq \frac{(L-1) \bar{u}\left(\xi_{i}\right)}{\left(1+h^{\prime}\left(x_{i}\right)+L \bar{u}\left(\xi_{i}\right)\right)\left(1+h^{\prime}\left(x_{i}\right)+\bar{u}\left(\xi_{i}\right)\right)}+\bar{u}\left(\xi_{i}\right) \\
& \quad \leq L \bar{u}\left(\xi_{i}\right)-\left(L^{2}-1\right) \bar{u}\left(\xi_{i}\right)^{2}+\mathcal{O}\left(x_{i}^{3}\right) \\
& \quad \leq L \bar{u}\left(\xi_{i+1}\right)+\sqrt{3 b}\left|y_{i+1}\right|-\left(L^{2}-1\right) \bar{u}\left(\xi_{i}\right)^{2}+\mathcal{O}\left(x_{i}^{3}\right) \\
& \quad \leq L \bar{u}\left(\xi_{i+1}\right),
\end{aligned}
$$

${ }^{10}$ Note that this computation holds for all $\xi_{i} \in Q_{\sqrt{\varrho}} \backslash P_{M}$. 
provided $\xi_{i+1} \notin P_{M}$, with $M \leq \sqrt{3 b}\left(L^{2}-1\right)$, which is fine provided $\varrho$ is chosen small enough. The above discussion can be summarized as follows.

Lemma 4.4. There exists $\varrho>0:$ For $\xi \notin Q_{2 \sqrt{\frac{b}{3}} \varrho}$ the unstable distribution is contained in $\mathcal{C}_{0}$. In addition, in the set $\left\{\xi=(x, y) \in Q_{\sqrt{\varrho}} \backslash\left(P_{M} \cup Q_{2 \sqrt{\frac{b}{3}} \varrho}\right): x y \leq 0\right\}$ the unstable direction is contained in $\mathcal{C}_{1}$.

To conclude we need to study what happens in a neighborhood of the origin of order $\varrho$. It is necessary to distinguish two possibilities: one can enter below the stable manifold, and hence be confined in the fat sector, or one can enter above the stable manifold, thereby being bound to the thin sector. We will start with the easy case: the second.

4.3. The cone field-Thin sector. If $x>0$, as soon as the trajectory, at some time $n$, enters $Q_{2 \sqrt{\frac{b}{3}} \varrho}$ we have that $(1, u) \in \mathcal{C}_{0}$ implies $u \geq 2 b \varrho \geq \gamma_{u}^{\prime}\left(x_{n}\right)$. Let us consider in $Q_{2 \sqrt{\frac{b}{3}} \varrho}$ the cone field $\mathcal{C}_{2}:=\{(1, u) \mid L \bar{u}(\xi) \geq$ $\left.u(x) \geq \gamma_{u}^{\prime}(x)\right\}$. Note that, upon entering in $Q_{2 \sqrt{\frac{b}{3}} \varrho}$ such a cone contains $\mathcal{C}_{1} \cdot{ }^{11}$ Now

$$
F(x, u) \geq F\left(x, \gamma_{u}^{\prime}(x)\right)=\gamma_{u}^{\prime}\left(x+h(x)+\gamma_{u}(x)\right) \geq \gamma_{u}^{\prime}(x+h(x)+y),
$$

where we have use that $\gamma_{u}^{\prime \prime}(x) \geq 0$ for $x \in[0, \varrho]$, provided $\varrho$ has been chosen small enough.

Lemma 4.5. In the region $Q_{2 \sqrt{\frac{b}{3}} \varrho} \backslash\left(P_{M} \cup\left\{\xi=(x, y) \in \mathbb{T}^{2}: y \geq \gamma^{u}(x)\right.\right.$ for $x>0 ; y \leq \gamma^{u}(x)$ for $\left.\left.x<0\right\}\right)$ the unstable direction is contained in the cone field $\mathcal{C}_{2}$.

Note that the above lemma suffices for trajectories in the thin sector. The situation it is not so simple in the fat sector since the lower bound would deteriorate to zero. A more detailed analysis is needed.

For each $\xi=(x, y) \in \mathbb{T}^{2}$, for which the unstable direction is defined, let $(1, u(\xi))$ be the vector in the unstable direction. Define then $\lambda_{u, n}(\xi, u)$ and $F_{n}(\xi, u)$ as in formulae (3.11) and (3.12) and similarly define the stable quantities. That is

$$
\begin{aligned}
& D_{T^{-n} \xi} T^{n}(1, u)=: \lambda_{u, n}(\xi, u)\left(1, F_{n}(\xi, u)\right) \\
& D_{\xi} T^{-n}(1,-v)=: \mu_{s, n}(\xi, v)\left(1,-F_{n}^{-}(\xi, v)\right)
\end{aligned}
$$

4.4. The cone field-Fat sector. First of all notice that the trajectory can enter $Q_{2 \sqrt{\frac{b}{3}} \varrho}$ either in $P_{M}$ or outside. Since the cone field $\mathcal{C}_{2}$ for $x \geq 0, \xi \notin P_{M}$ contains the unstable vector (Lemma 4.5), we have a good control on the unstable vector in both cases until we enter in $P_{M}$. Upon entering $P_{M}$, we will obtain a very sharp control on the evolution of the edges of the cone. Let $\xi \notin P_{M}, T \xi \in P_{M}$, and let $\ell_{+}-1>0$ be the smallest integer such that $\xi_{n} \notin P_{M}$. By equation (3.11), we have

$$
u_{n}:=F_{n}\left(\xi_{n}, u\right)=\sum_{i=1}^{n} \lambda_{u, i}\left(\xi_{n-i}, u_{n-i}\right)^{-1} h^{\prime}\left(x_{n-i}\right)+\lambda_{u, n}\left(\xi_{n}, u\right)^{-1} u .
$$

Then, for each $n<\ell_{+}$, holds true

$$
u_{n} \leq \sum_{i=1}^{n} h^{\prime}\left(x_{n-i}\right)+u \leq \frac{\mathrm{C}}{M}\left|\sum_{i=1}^{n} y_{i}\right|+u .
$$

By Lemma 4.3. (3),(5), it follows that we have, for $u \in \mathcal{C}_{2}(\xi)$,

$$
u_{n} \leq \mathrm{C}_{+} \sqrt{\left|y_{n}\right|}
$$

Moreover, remembering (3.11) and that $u \in \mathcal{C}_{2}(\xi)$, yields

$$
u_{n} \geq e^{-2 n \mathrm{C}_{+} \sqrt{\left|y_{n}\right|}} u \geq \mathrm{C} u \geq \mathrm{C}_{-} \sqrt{\left|y_{n}\right|} .
$$

Consequently, if for $\xi=(x, y)$ we define the cone $\mathcal{C}_{3}(\xi)=\left\{\mathrm{C}_{-} \sqrt{|y|} \leq u \leq \mathrm{C}_{+} \sqrt{|y|}\right\}$., then the above results can be written as follows.

Lemma 4.6. In $P_{M}$ the unstable direction is contained in the cone field $\mathcal{C}_{3}$.

\footnotetext{
${ }^{11}$ Note that, in such a case, the trajectory cannot enter in $P_{M}$.
} 
Finally we have to follow the trajectory outside $P_{M}$ until it exits from $Q_{2 \sqrt{\frac{b}{3}} \varrho}$. The upper bound can be treated as before. Not so for the lower bound.

Let $\xi=(x, y)$ be a point in the fat sector, $x \leq 0, x_{-1} \geq 0$. Then, remembering subsection 4.1 let $E:=H(x, y), u_{0}=0$ and $u_{n+1}:=F\left(x_{n}, u_{n}\right)$. Clearly, $D_{(x, y)} T^{n} \mathcal{C}_{+} \subset\left\{(1, u) \in \mathbb{R}^{2} \mid u \geq u_{n}\right\} \cup\{0\}$.

Lemma 4.7. In the situation described above, for each $n \in \mathbb{N}$, holds true

$$
F\left(x_{n}, \Upsilon_{E}^{\prime}\left(x_{n}\right)\right)-\Upsilon_{E}^{\prime}\left(x_{n+1}\right)=\mathcal{O}\left(\left|y_{n}\right|^{3 / 2}\right)
$$

Proof. Notice that, since the trajectory lies below the unstable manifold, $|y| \geq \mathrm{C} x^{2}$. It is then convenient to keep track of the orders of magnitude only in terms of powers of $y$.

$$
F\left(x_{n}, \Upsilon_{E}^{\prime}\left(x_{n}\right)\right)=\Upsilon_{E}^{\prime}\left(x_{n}\right)-\Upsilon_{E}^{\prime}\left(x_{n}\right)^{2}+h^{\prime}\left(x_{n}\right)+\mathcal{O}\left(\left|y_{n}\right|^{3 / 2}\right)
$$

On the other hand, differentiating (4.1), one gets

$$
\Upsilon_{E}^{\prime}(x)=\frac{h(x)}{\Upsilon_{E}(x)}-\frac{h(x)^{2}}{2 \Upsilon_{E}(x)^{2}}-\frac{h^{\prime}(x)}{2}+\mathcal{O}\left(\left|\Upsilon_{E}\right|^{3 / 2}\right) .
$$

Accordingly, by (4.2),

$$
\begin{aligned}
\Upsilon_{E}^{\prime}\left(x_{n+1}\right) & =\frac{h\left(x_{n}\right)+h^{\prime}\left(x_{n}\right) \Upsilon_{E}\left(x_{n}\right)}{\Upsilon_{E}\left(x_{n}\right)+h\left(x_{n}\right)}-\frac{h\left(x_{n}\right)^{2}}{2 \Upsilon_{E}\left(x_{n}\right)^{2}}-\frac{h^{\prime}\left(x_{n}\right)}{2}+\mathcal{O}\left(\left|y_{n}\right|^{3 / 2}\right) \\
& =\Upsilon_{E}^{\prime}\left(x_{n}\right)-\Upsilon_{E}^{\prime}\left(x_{n}\right)^{2}+h^{\prime}\left(x_{n}\right)+\mathcal{O}\left(\left|y_{n}\right|^{3 / 2}\right),
\end{aligned}
$$

from which the Lemma easily follows.

Since $F$ is a contraction in $u$, we can estimate

$$
\begin{aligned}
\left|u_{n}-\Upsilon_{E}^{\prime}\left(x_{n}\right)\right| & =\left|F\left(x_{n-1}, u_{n-1}\right)-F\left(x_{n-1}, \Upsilon_{E}^{\prime}\left(x_{n-1}\right)\right)\right|+\mathcal{O}\left(\left|y_{n-1}\right|^{3 / 2}\right) \\
& \leq\left|u_{n-1}-\Upsilon_{E}^{\prime}\left(x_{n-1}\right)\right|+\mathcal{O}\left(\left|y_{n-1}\right|^{3 / 2}\right) \\
& \leq\left|\Upsilon_{E}^{\prime}\left(x_{0}\right)\right|+\mathcal{O}\left(\sum_{k=0}^{n-1}\left|y_{k}\right|^{3 / 2}\right) \\
& =\mathcal{O}\left(\left|y_{0}\right|+\sum_{k=0}^{n-1} \sqrt{\left|y_{k}\right|}\left(x_{k}-x_{k+1}\right)\right)=\mathcal{O}\left(\left|y_{n}\right|\right) .
\end{aligned}
$$

We have thus proved that there exists a constant $\mathrm{C}_{0}>0$ such that

$$
u_{n} \geq \Upsilon_{E}^{\prime}\left(x_{n}\right)-\mathrm{C}_{0} \Upsilon_{E}\left(x_{n}\right)
$$

Hence outside $P_{M}$ the image of the cone will belong to the cone field $\mathcal{C}_{3}:=\left\{(1, u) \in \mathbb{R}^{2} \mid u(\xi) \geq \Upsilon_{E(\xi)}^{\prime}(x)-\right.$ $\left.\mathrm{C}_{0} \Upsilon_{E(\xi)}(x)\right\}$. Note that, upon exiting $P_{M}, \Upsilon_{E}^{\prime}\left(x_{n}\right)-\mathrm{C}_{0} \Upsilon_{E}\left(x_{n}\right) \geq \mathrm{C}_{-}^{\prime} \sqrt{\left|y_{n}\right|}$, provided $\varrho$ is chosen small enough. The Proposition follows by choosing $\varrho$ small enough and remembering Lemmata 4.44 .5 and 4.6

\section{AN A PRIORI EXPANSION BOUND}

The results of the previous section allow to obtain the following nice estimate on the expansion in the system.

Lemma 5.1. There exists $K>0$ such that, for each $\xi=(x, y) \in \mathbb{T}^{2} \backslash\{0\}, n \in \mathbb{N}$ and $(1, u) \in \mathcal{C}_{*}\left(T^{-n} \xi\right)=$ $\mathcal{C}_{*}\left(\xi_{-n}\right)$, holds true

$$
\lambda_{u, n}(\xi, u) \geq e^{-K|x|}\left(K^{-1}|x| n+1\right)^{2} .
$$

Proof. Let us fix $\delta>0$. On the one hand, if the trajectory lies outside of $Q_{\delta}$, then we have an exponential expansion, on the other hand, if the backward trajectory enjoys $\left|x_{-n}\right| \geq\left|x_{0}\right|$, then equation (3.11) and Proposition 4.1 imply

$$
\lambda_{u, n}(\xi, u) \geq\left(1+K_{-}\left|x_{0}\right|\right)^{n} \geq e^{-K\left|x_{0}\right|}\left(K^{-1}\left|x_{0}\right| n+1\right)^{2} .
$$

We say that the backward orbit of $\xi$ (up to time $n$ ) passes $p$ times thru $Q_{\delta}$ if $\left\{0 \leq k \leq n: \xi_{-k} \in Q_{\delta}\right\}$ consists of $p$ intervals. The Lemma holds for orbits that pass zero-times thru $Q_{\delta}$. Suppose it holds for orbits 
that pass $p$ times. Let $\xi_{-n} \in Q_{\delta}$ and let $m<n$ be the last time $\xi_{-m} \notin Q_{\delta}$ but it passed already $p$ times in $Q_{\delta}$. Moreover, suppose that the Lemma holds in $Q_{2 \delta}$. Accordingly, for each $n \geq l$ such that $\xi_{-n} \in Q_{\delta}$ holds

$$
\begin{aligned}
\lambda_{u, n}(\xi, u) & \geq e^{-K\left|x_{0}\right|}\left(K^{-1}\left|x_{0}\right| m+1\right)^{2} e^{-2 K \delta}\left(2 K^{-1} \delta(n-m)+1\right)^{2} \\
& \geq e^{-K\left|x_{0}\right|}\left(K^{-1}\left|x_{0}\right| m+1\right)^{2}\left(K^{-1} \delta(n-m)+1\right)^{2} \\
& \geq e^{-K\left|x_{0}\right|}\left(K^{-1}\left|x_{0}\right| n+1\right)^{2},
\end{aligned}
$$

provided $\delta$ has been chosen small enough and since it must be $n-m \geq \mathrm{C} \delta^{-1}$. Thus to prove the Lemma it suffices to prove it for the pieces of trajectories in $Q_{\delta}$. There are two cases: a trajectory enters in the thin sector or in the fat one. Let us consider the thin sector first. Set $u_{-j}:=F_{n-j}\left(\xi_{-j}, u\right)$. By the usual distortion estimates follows

$$
\begin{aligned}
\lambda_{u, n}(\xi, u) & =\prod_{j=1}^{n}\left(1+h^{\prime}\left(x_{-j}\right)+u_{-j}\right) \geq \prod_{j=1}^{n}\left(1+h^{\prime}\left(x_{-j}\right)+\gamma_{u}^{\prime}\left(x_{-j}\right)\right) \\
& \geq \prod_{j=1}^{n}\left(1+h^{\prime}\left(f_{u}^{-j}(x)\right)+\gamma_{u}^{\prime}\left(f_{u}^{-j}(x)\right)\right)=\prod_{j=1}^{n}\left(f_{u}\right)^{\prime}\left(f_{u}^{-j}(x)\right) \\
& \geq \prod_{j=1}^{n} \frac{\left|f_{u}^{-j+1}(x)-f_{u}^{-j}(x)\right|}{\left|f_{u}^{-j}(x)-f_{u}^{-j-1}(x)\right|} e^{-\mathrm{C}\left|f_{u}^{-j+1}(x)-f_{u}^{-j}(x)\right|} \\
& \geq e^{-\mathrm{C}\left|x_{0}\right|} \frac{\left|x_{0}\right|^{2}}{\left|f_{u}^{-n}\left(x_{0}\right)\right|^{2}} .
\end{aligned}
$$

Now, notice that $f_{u}^{-1}(x) \leq \frac{x}{1+\mathrm{C} x}$, hence $f_{u}^{-n}\left(x_{0}\right) \leq \frac{\left|x_{0}\right|}{1+\mathrm{C} n\left|x_{0}\right|}$. Thus,

$$
\lambda_{u, n}(\xi, u) \geq e^{-\mathrm{C}\left|x_{0}\right|}\left(1+n \mathrm{C}\left|x_{0}\right|\right)^{2} .
$$

For the fat sector we need only to consider the cases in which $x_{0} \notin Q_{\delta}$ and $x_{0} \in Q_{\delta}, x_{0} \leq 0$ since if $x_{0}>0$ the backward trajectory increases the $x$ coordinate. In such cases we have ${ }^{12}$

$$
\begin{aligned}
\lambda_{u, n}(\xi, u) & =\prod_{j=1}^{n}\left(1+h^{\prime}\left(x_{-j}\right)+u_{-j}\right) \geq \prod_{j=1}^{n}\left(1+\Upsilon_{E}^{\prime}\left(x_{-j}\right)-\mathrm{C}_{0} \Upsilon_{E}\left(x_{-j}\right)\right) \\
& \geq e^{\sum_{j=1}^{n} \Upsilon_{E}^{\prime}\left(x_{-j}\right)-2 \mathrm{C}_{0} \Upsilon_{E}\left(x_{-j}\right)} \\
& \geq e^{-\sum_{j=1}^{n} \frac{\Upsilon_{E}^{\prime}\left(x_{-j}\right)}{\Upsilon_{E}\left(x_{-j}\right)}\left(x_{-j-1}-x_{-j}\right)-3 \mathrm{C}_{0} \sum_{j=1}^{n}\left(x_{-j-1}-x_{-j}\right)} \\
& \geq e^{-\mathrm{C}\left|x_{0}\right|} e^{-\int_{x_{0}}^{x} \frac{\Upsilon_{E}^{\prime}(z)}{\Upsilon_{E}(z)} d z}=e^{-\mathrm{C}\left|x_{0}\right|} \frac{\Upsilon_{E}\left(x_{0}\right)}{\Upsilon_{E}\left(x_{-n}\right)}
\end{aligned}
$$

Let $n_{*} \in \mathbb{N}$ be the last integer for which $\left|G\left(x_{-n}\right)\right| \geq E$, then for $n \leq n_{*}$ we have

$$
\lambda_{u, n}(\xi) \geq e^{-\mathrm{C}\left|x_{0}\right|} \sqrt{\frac{E+G\left(x_{0}\right)}{E+G\left(x_{-n}\right)}} \geq e^{-\mathrm{C}\left|x_{0}\right|} \sqrt{\frac{G\left(x_{-n}\right)+G\left(x_{0}\right)}{2 G\left(x_{-n}\right)}} .
$$

On the other hand comparing the backward motion with the backward motion on the stable manifold, as we did before with the unstable, ${ }^{13}$

$$
\lambda_{u, n}(\xi, u) \geq e^{-\mathrm{C}\left|x_{0}\right|} \sqrt{\frac{1+\left(n\left|x_{0}\right| \mathrm{C}+1\right)^{4}}{2}} \geq e^{-\mathrm{C}\left|x_{0}\right|}\left(1+n\left|x_{0}\right| \mathrm{C}\right)^{2} .
$$

\footnotetext{
${ }^{12}$ Again, $E$ is chosen to be the energy associated to the point of the orbit closer to the origin.

${ }^{13}$ Here we use the inequality

$$
\sqrt{\frac{1+(1+a)^{4}}{2}} \geq\left(1+\frac{a}{2}\right)^{2}
$$
}


Next, let us consider $n \in\left\{n_{*}, \ldots, m\right\}$, where $m$ is the larger integer such that $x_{-m} \leq 0$, we have $2 \sqrt{E} \geq$ $\Upsilon_{E}\left(x_{-n}\right) \geq \sqrt{2 E}$.

$$
\begin{aligned}
\lambda_{u, n-n_{*}}(\xi, u) & \geq e^{-\mathrm{C}_{2}\left|x_{n_{*}}\right|} e^{-\int_{x_{-n}}^{x_{-}} \frac{\Upsilon_{E}^{\prime}(z)}{\Upsilon_{E}(z)} d z} \geq e^{-\mathrm{C}\left|x_{0}\right|} \frac{\Upsilon_{E}\left(x_{-n_{*}}\right)}{\Upsilon_{E}\left(x_{-n}\right)} \\
& \geq e^{-\mathrm{C}\left|x_{0}\right|} \sqrt{\frac{3}{2}} \geq e^{-\mathrm{C}\left|x_{0}\right|}\left(1+\mathrm{C}\left|x_{-n_{*}}\right|\left(n-n_{*}\right)\right)^{2},
\end{aligned}
$$

where, in the last line, we used Lemma 4.3.(5). By symmetry it will be enough to wait another time $m$ to have $\left|x_{-2 m}\right| \geq \frac{1}{2}\left|x_{0}\right|$, after which the expansion is assured by the estimate (5.1).

Next we need to have similar estimates for the stable contraction. By (4.4)

$$
\begin{aligned}
\mu_{s}(v) & :=1+v=\mu_{s, 1}(\xi, v) \\
F_{1}^{-}(\xi, v) & =h^{\prime}(x-y)+\frac{v}{1+v} .
\end{aligned}
$$

It is immediate to check that $D_{(x, y)} T^{-1}(1,-v)=\mu_{s}(v)\left(1,-F_{1}^{-}((x, y), v)\right)$ and $\mu_{s, n}\left(\xi, v_{0}\right):=\prod_{i=0}^{n-1} \mu_{s}\left(v_{-i}\right)$, where $v_{0}=v$ and $v_{-i-1}:=F_{1}^{-}\left(T^{-i} \xi, v_{-i}\right)$.

An interesting way to transform information on expansion into information on contraction is to use area preserving.

Lemma 5.2. Let $\xi \in \mathbb{T}^{2}$, then for each $n \in \mathbb{N}, u, v \geq 0$ let $u_{-n}=u, v_{0}=v, \xi_{-n}=T^{-n} \xi$ and $u_{-k+1}=$ $F\left(\xi_{-k+1}, u_{-k}\right), v_{-k-1}=F^{-}\left(\xi_{-k}, v_{-k}\right)$. Then

$$
\mu_{s, n}\left(\xi, v_{0}\right)\left(v_{-n}+u_{-n}\right)=\lambda_{u, n}\left(\xi, u_{-n}\right)\left(u_{0}+v_{0}\right) .
$$

Proof. Calling $\omega$ the standard symplectic form we have

$$
\begin{aligned}
\mu_{s, n}\left(\xi, v_{0}\right)\left(v_{-n}+u_{-n}\right) & =\omega\left(D_{\xi} T^{-n}\left(1,-v_{0}\right),\left(1, u_{-n}\right)\right) \\
& =\omega\left(\left(1,-v_{0}\right), D_{\xi_{-n}} T^{n}\left(1, u_{-n}\right)\right)=\lambda_{u, n}\left(\xi, u_{-n}\right)\left(u_{0}+v_{0}\right) .
\end{aligned}
$$

The following is an immediate corollary of Lemmata 5.2 and 5.1

Corollary 5.3. For each $\xi=(x, y) \in \mathbb{T}^{2}$ and $n \in \mathbb{N}$ holds

$$
\mu_{s, n}\left(\xi, v_{0}\right) \geq e^{-\mathrm{C}\left|x_{0}\right|}\left(\mathrm{C}^{-1}\left|x_{0}\right| n+1\right)^{2} \frac{u_{0}+v_{0}}{u_{-n}+v_{-n}} \quad \forall n \in \mathbb{N} .
$$

All the other expansion estimates can be obtained by reversibility.

\section{Distributions-Regularity}

Let $(1, u(\xi)),(1,-v(\xi))$ be the unstable and stable directions, respectively. We will then use the short hand $\lambda_{u, n}(\xi):=\lambda_{u, n}\left(\xi, u\left(\xi_{-n}\right)\right)$ and $\mu_{s, n}(\xi):=\mu_{s, n}\left(\xi, v\left(\xi_{n}\right)\right)$.

Lemma 6.1. The unstable distribution is continuous in $\mathbb{T}^{2}$.

Proof. Notice that, for $\xi=(x, y), \xi_{n}:=T^{n} \xi$, iterating formula (3.11), in analogy with (3.12), holds true

$$
\begin{aligned}
u(x)-u(z)= & \lambda_{u, n}(x)^{-1} \lambda_{u, n}(z)^{-1}\left(u\left(x_{-n}\right)-u\left(z_{-n}\right)\right) \\
& +\sum_{k=1}^{n} \lambda_{u, k}(x)^{-1} \lambda_{u, k}(z)^{-1}\left(h^{\prime}\left(x_{-k}\right)-h^{\prime}\left(z_{-k}\right)\right)
\end{aligned}
$$

By Lemma 5.1] we can take the limit $n \rightarrow \infty$ in the above formula provided $x \neq 0$, and obtain a uniformly convergent series from which the continuity follows. If $\xi \neq 0$ then $x_{-1} \neq 0$ and (3.11) implies

$$
u(\xi)=\lambda_{u}\left(\xi_{-1}\right)^{-1} h^{\prime}\left(x_{-1}\right)+\lambda_{u}\left(\xi_{-1}\right)^{-1} u\left(\xi_{-1}\right),
$$

hence the continuity at $\xi \neq 0$ follows. We are left with the continuity at the origin, but this is already implied by Proposition 4.1 
This means that we can extend the invariant unstable distribution (that, up to now, where defined-by Pesin theory-only almost everywhere) to a continuous everywhere defined vector field. The same statement holds for the stable vectors by reversibility.

Given a continuous vector field there exists integral curves. Since we do not know yet if the vector fields are Lipschitz, it does not follows automatically that from a given point there exits only one integral curve, yet this follows by standard dynamical arguments. Clearly such integral curves are nothing else than the stable and unstable manifolds that are therefore everywhere defined. In addition, remember that, by general hyperbolic theory, the foliations are absolutely continuous, it follows that the above everywhere defined foliations are continuous. Unfortunately, for the following much sharper regularity information is needed, this is obtained in the rest of the section.

Let us call $\partial^{u}, \partial^{s}$ the derivative along the unstable and the stable vector fields, respectively.

Lemma 6.2. The vector field $u$ is $\mathcal{C}^{1}$ along the unstable manifolds, apart from the origin, moreover

$$
\left|\partial^{u} u(\xi)\right| \leq C \quad \forall \xi \neq 0 .
$$

Proof. If $\xi$ is outside of a neighborhood of the origin of size $\delta$, then by Lemma 5.1] (6.1) we have, in analogy with the arguments leading to (3.13),

$$
\left|\partial^{u} u(\xi)\right|=\left|\sum_{k=1}^{\infty} \lambda_{u, k}(x)^{-3} h^{\prime \prime}\left(x_{k}\right)\right| \leq \mathrm{C} \sum_{n=0}^{\infty}(\delta n+1)^{-6} \leq \mathrm{C} .
$$

Since the series converges uniformly the $\mathcal{C}^{1}$ property follows. To obtain a uniform bound more work is needed. If $|\xi|<\delta$, formula (6.2) implies

$$
\left|\partial^{u} u(\xi)\right| \leq \lambda_{u}\left(\xi_{-1}\right)^{-3}\left|h^{\prime \prime}\left(x_{-1}\right)\right|+\lambda_{u}\left(\xi_{-1}\right)^{-3}\left|\partial^{u} u\left(\xi_{-1}\right)\right|=: \Psi\left(\xi_{-1},\left|\partial^{u} u\left(\xi_{-1}\right)\right|\right) .
$$

A simple computation, remembering Proposition 4.1] shows that

$$
\Psi(\xi, \varrho) \leq 7 b|x|+\frac{\varrho}{1+3|u(\xi)|} \leq 7 b|x|+\frac{\varrho}{1+3 K_{-}|x|} \leq \varrho,
$$

provided $\varrho \geq \frac{7 b\left(1+3 K_{-}\right)}{3 K_{-}}$. Accordingly, for $\rho$ large enough, we have $\left|\partial^{u} u(\xi)\right| \leq \rho$, for all $\xi$.

It remains to investigate the regularity of the unstable distribution along the stable direction.

Lemma 6.3. The unstable distributions are $\mathcal{C}^{1}$ along stable manifolds, apart from the origin. Moreover

$$
\left|\partial^{s} u(\xi)\right| \leq \mathrm{C} \quad \forall \xi \neq 0 .
$$

Proof. Let us fix some arbitrary neighborhood of the origin. Let $x, z \in W^{s}$ outside such a neighborhood. Let $W_{0}^{s}$ be the piece of stable manifold between such two points. Clearly $W_{n}^{s}:=T^{n} W_{0}^{s}$ grows for negative $n$. Let $n(x, z)$ be the largest integer for which $\left|W_{-n}^{s}\right| \leq\left|W_{0}^{s}\right|^{\frac{1}{4}}$. Our first result is a distortion bound.

Sub-lemma 6.4. For each $n \leq n(x, z)$ and $\xi \in W_{0}^{s}$, holds

$$
\mathrm{C}^{-1} \frac{\left|W_{-n}^{s}\right|}{\left|W_{0}^{s}\right|} \leq \mu_{s, n}(\xi) \leq \mathrm{C} \frac{\left|W_{-n}^{s}\right|}{\left|W_{0}^{s}\right|}
$$

Proof. If the backward orbit spends at least half of the time outside the neighborhood, then $W_{-n}^{s}$ grows exponentially fast, hence $n(x, z) \leq \mathrm{C} \ln \left|W_{0}^{s}\right|^{-1}$ and $\sum_{i=0}^{n(x, z)}\left|W_{-i}^{s}\right| \leq \mathrm{C}$. If this is not the case, the worst possible situation is when $W_{-m}^{s}$ is the closest to the origin and all the trajectory lies in the neighborhood. In such a case, letting $m:=n(x, z)$,

$$
\left|W_{m}^{s}\right|=\int_{W_{0}^{s}} \mu_{s, m}(z) d z \geq \int_{W_{0}^{s}} \frac{\theta(z)\left(\mathrm{C}^{-1}|z| m+1\right)^{2}}{2 \theta\left(T^{-m} z\right)} d z
$$

where $\theta(\zeta)=u(\zeta)+v(\zeta)$ is the separation between the stable and the unstable directions at the point $\zeta$ and we have used Lemma 5.3 Now Proposition 4.1 and Lemma 4.3 (1) imply $\theta\left(T^{-m} z\right) \leq \mathrm{C} \mathrm{m}^{-1}$ outside the parabolic sector, while Lemma $4.3 .(3,4,5)$ show that the same estimates remain in $P_{M}$ as well. Accordingly,

$$
\left|W_{-m}^{s}\right| \geq \mathrm{C} m^{3}\left|W_{0}^{s}\right| \text {. }
$$


That is $m \leq \mathrm{C}\left|W_{0}^{s}\right|^{-\frac{1}{4}}$, and

$$
\sum_{i=0}^{n(x, z)}\left|W_{-i}^{s}\right| \leq n(x, z)\left|W_{0}^{s}\right|^{\frac{1}{4}} \leq \mathrm{C} .
$$

The above estimate readily implies that, for each $\xi, \eta \in W_{s}^{0}$,

$$
e^{-\mathrm{C}\left|W_{-i}^{s}\right|} \leq \frac{\mu_{s}\left(\xi_{-i}, v\left(\xi_{-i}\right)\right)}{\mu_{s}\left(\eta_{-i}, v\left(\eta_{-i}\right)\right)} \leq e^{\mathrm{C}\left|W_{-i}^{s}\right|}
$$

where we have used Lemma 6.2 for the stable manifold. Accordingly,

$$
e^{-\mathrm{C} \sum_{i=0}^{m}\left|W_{-i}^{s}\right|} \leq \frac{\mu_{s, n}(\xi)}{\mu_{s, n}(\eta)} \leq e^{\mathrm{C} \sum_{i=0}^{m}\left|W_{-i}^{s}\right|},
$$

from which the Lemma readily follows.

By Lemma 5.2 it follows, letting again $m:=n(x, z)$,

$$
\begin{aligned}
\lambda_{u, m}(x)^{-1} \lambda_{u, m}(z)^{-1} & =\lambda_{u, m}(x)^{-1} \sqrt{\lambda_{u, m}(z)^{-2}} \\
& \leq \mathrm{C}\left(\theta\left(x_{-m}\right) \mu_{s, m}(x) \sqrt{\lambda_{u, m}(z) \theta\left(z_{-m}\right) \mu_{s, m}(z)}\right)^{-1}
\end{aligned}
$$

As before the worst case is clearly when $W_{-m}^{s}$ is the closest to the origin. In such a case, consider that at least one of the two end points of $W_{-n(x, z)}^{s}$ must be at a distance $\mathrm{C}\left|W_{-n(x, z)}^{s}\right|$ from the fixed point, let us say $T^{-n(x, z)} z$, hence $\theta\left(T^{-n(x, z)} z\right) \geq \mathrm{C}\left|W_{-n(x, z)}^{s}\right|$. In addition, $\theta\left(x_{-m}\right) \geq \mathrm{C} m^{-1}$. Indeed, this follows from Lemma 4.3 $(3,4,5)$ if the trajectory ends in $P_{M}$. If the trajectory lies outside $P_{M}$ then it approaches the origin slower than the dynamics $x-\mathrm{C} x^{2}$, which implies $x_{-m} \geq \mathrm{Cm}^{-1}$. Furthermore by using the above facts, Lemma 5.1. Sub-lemma 6.4 and the definition of the stopping time $m$ yields

$$
\lambda_{u, m}(x)^{-1} \lambda_{u, m}(z)^{-1} \leq \mathrm{C}|x-z|^{\frac{3}{4}} m \frac{1}{m} \sqrt{\frac{|x-z|^{\frac{3}{4}}}{|x-z|^{\frac{1}{4}}}} \leq \mathrm{C}|x-z| .
$$

Since we know that $u$ is a uniformly continuous function it follows

$$
\lim _{z \rightarrow x} \lambda_{u, m}(x)^{-1} \lambda_{u, m}(z)^{-1} \frac{\left|u\left(T^{-m} x\right)-u\left(T^{-m} z\right)\right|}{|x-z|}=0 .
$$

Accordingly, by formula (3.12),

$$
\begin{aligned}
u^{\prime}(x) & =\lim _{z \rightarrow x} \sum_{n=0}^{m} \lambda_{u, n}(x)^{-1} \lambda_{u, n}(z)^{-1} \frac{h^{\prime}\left(T^{-n} x\right)-h^{\prime}\left(T^{-n} z\right)}{x-z} \\
& =\lim _{z \rightarrow x} \sum_{n=0}^{m} \lambda_{u, n}(x)^{-1} \lambda_{u, n}(z)^{-1} h^{\prime \prime}\left(T^{-n} \zeta_{n}\right) \mu_{s, n+1}\left(\zeta_{n}\right),
\end{aligned}
$$

for some $\zeta_{n} \in W_{0}$. But $\left|h^{\prime \prime}\left(T^{-n} \zeta_{n}\right)\right| \leq \mathrm{C} \theta\left(T^{-n} z\right)$, hence

$$
\begin{aligned}
& \lambda_{u, n}(x)^{-1} \lambda_{u, n}(z)^{-1} \theta\left(T^{-n} x\right) \mu_{s, n+1}(x) \leq \mathrm{C} \lambda_{u, n}(z)^{-1} \\
& \lambda_{u, n}(x)^{-1} \lambda_{u, n}(z)^{-1} \theta\left(T^{-n} z\right) \mu_{s, n+1}(z) \leq \mathrm{C} \lambda_{u, n}(x)^{-1} .
\end{aligned}
$$

Remembering Sub-Lemma 6.4 the uniform convergence of the series follows and yields the formula

$$
\partial^{s} u(x)=\sum_{n=0}^{\infty} \lambda_{u, n}(x)^{-2} \mu_{s, n+1}(x) h^{\prime \prime}\left(T^{-n} x\right) .
$$


Given the arbitrariness of the neighborhood of zero, the above formula holds for each $x \neq 0$ and, since the series converges uniformly, the $\mathcal{C}^{1}$ property follows. We can now conclude the Lemma. By Lemma 5.2 follows

$$
\begin{aligned}
\left|\partial^{s} u(x)\right| & \leq \mathrm{C} \sum_{n=0}^{\infty} \lambda_{u, n}(x)^{-2} \mu_{s, n}(x) \theta\left(T^{-n} x\right) \\
& \leq \mathrm{C} \sum_{n=0}^{\infty} \lambda_{u, n}(x)^{-1} \theta(x) \leq \mathrm{C} \sum_{n=0}^{\infty} \frac{|x|}{(|x| n+1)^{2}} \leq \mathrm{C} .
\end{aligned}
$$

Remark 6.5. Notice that the symmetrical statements follow by reversibility.

The final result on the regularity of the foliations can be stated as follows.

Lemma 6.6. The stable and unstable vector fields are $\mathcal{C}^{1}\left(\mathbb{T}^{2} \backslash\{0\}\right)$ and, more precisely, for each $\xi \in \mathbb{T}^{2} \backslash\{0\}$,

$$
|D u(\xi)| \leq \mathrm{C} \theta(\xi)^{-1} .
$$

Proof. The $\mathcal{C}^{1}$ property follows from Lemma 19.1.10 of [7]. Then the size of the derivative can be easily estimated by the size of the partial derivatives in the stable and unstable directions divided by the angle between them.

Remark 6.7. In fact, it is likely that with a little more work one can show that the foliations are $\mathcal{C}^{\frac{3}{2}-\varepsilon}$, but we do not investigate this possibility since it is not needed in the following.

\section{HOLONOMY}

There exists $\mathrm{C}_{1}>0$ such that, given two close by stable manifolds $W_{1}^{s}, W_{2}^{s}$ we can define the unstable holonomy $\Psi^{u}: W_{1}^{s} \rightarrow W_{2}^{s}$ by $\left\{\Psi^{u}(\xi)\right\}:=W^{u}(\xi) \cap W_{2}^{s}$. Let $D_{r}:=\left\{\zeta=\left(z_{1}, z_{2}\right) \in \mathbb{R}^{2}:\left|z_{1}\right| \leq r ;\left|z_{2}\right| \leq r^{2}\right\}$.

Lemma 7.1. For each $W_{1}^{s}, W_{2}^{s}$ disjoint from $D_{r}$ and $\xi \in W_{1}^{s}$, holds

$$
\left|J \Psi^{u}(\xi)-1\right| \leq \mathrm{C} r^{-1}\left\|\Psi^{u}(\xi)-\xi\right\| .
$$

Provided $\left\|\Psi^{u}(\xi)-\xi\right\| \leq \mathrm{C}_{1} r$.

Proof. Let $\gamma_{s}, \tilde{\gamma}_{s}:[-\delta, \delta] \rightarrow \mathbb{R}^{2}$ be $W_{1}^{s}, W_{2}^{s}$, respectively, parametrized by arc-length. Also, let $\Gamma:[-\delta, \delta]^{2} \rightarrow$ $\mathbb{R}^{2}$, be such that $\Gamma(0,0)=\xi, \Gamma(s, 0)=\gamma_{s}(s)$ and $\Gamma(s, t)$ be the unstable manifold, parametrized by arc-length, of $\Gamma(s, 0)$ and, finally, $\Gamma(0, \rho):=\Psi^{u}(\xi)$. Note that $\Gamma(s, t)$ can be obtained integrating the unstable vector field starting from $\Gamma(s, 0)$, hence Lemma 6.6 and the standard results on the continuity with respect to the initial data imply $\Gamma \in \mathcal{C}^{1}$. By the transversality of the stable and unstable manifolds there exist $\tau, \sigma:[-\delta, \delta] \rightarrow \mathbb{R}$ such that $\Gamma(s, \tau(s))=\Psi^{u}\left(\gamma_{s}(s)\right)=\tilde{\gamma}(\sigma(s)) \in W_{2}^{s}$. Calling $\eta(s)$ the unit vector perpendicular to $\tilde{\gamma}^{\prime}(s)$, by the implicit function theorem, it follows

$$
\begin{aligned}
\tau^{\prime}(s) & =-\frac{\left\langle\eta(s), \partial^{s} \Gamma(s, \tau(s))\right\rangle}{\left\langle\eta(s), \partial_{t} \Gamma(s, \tau(s))\right\rangle} \\
\sigma^{\prime}(s) & =\left\langle\tilde{\gamma}_{s}^{\prime}(s), \partial^{s} \Gamma(s, \tau(s))\right\rangle-\frac{\left\langle\tilde{\gamma}_{s}^{\prime}(s), \partial_{t} \Gamma(s, \tau(s))\right\rangle\left\langle\eta(s), \partial^{s} \Gamma(s, \tau(s))\right\rangle}{\left\langle\eta(s), \partial_{t} \Gamma(s, \tau(s))\right\rangle}
\end{aligned}
$$

where, clearly, $\sigma^{\prime}(s)=J \Psi^{u}\left(\gamma_{s}(s)\right)$. Calling $v^{u}(\eta), \eta \in \mathbb{T}^{2}$, the unit vector in the unstable direction at $\eta$ and $v^{s}(\eta)$ the stable one, one has $\partial_{t} \Gamma(s, \tau(s))=v^{u}(\Gamma(s, \tau(s)))$. On the other hand, setting $V(s, t):=$ $\partial^{s} \Gamma(s, t)-v^{s}(\Gamma(s, t))$, holds $V(s, t)=0$ for $t=0$, but for $t \neq 0$, in general, it will be $V(s, t) \neq 0$. Yet, it is possible to estimate it by differentiating $\Gamma(s, t)=\Gamma(s, 0)+\int_{0}^{t} v^{u}\left(\Gamma\left(s, t^{\prime}\right)\right) d t^{\prime}$ which yields

$$
\partial^{s} \Gamma(s, t)=v^{s}(\Gamma(s, 0))+\int_{0}^{t} D v^{u}\left(\Gamma\left(s, t^{\prime}\right)\right) \partial^{s} \Gamma\left(s, t^{\prime}\right) d t^{\prime} .
$$

Lemmata 6.6 and 6.3 imply that $\left\|D v^{u}\right\| \leq \mathrm{C} r^{-1}$ and $\left\|D v^{u} v^{s}\right\|=\left|\partial^{s} v^{u}\right| \leq \mathrm{C}$, hence

$$
\|V(s, t)\| \leq \mathrm{C} r^{-1} \int_{0}^{t}\left\|V\left(s, t^{\prime}\right)\right\| d t^{\prime}+\mathrm{C} t .
$$


By Gronwal, it follows, provided $t \leq \mathrm{C} \rho$ and $\rho \leq \mathrm{C}_{1} r$, for $\mathrm{C}_{1}$ small enough,

$$
\|V(s, t)\| \leq \mathrm{C} t
$$

Accordingly, by the second of (7.1) and (7.2), it follows

$$
\left|\sigma^{\prime}(0)-1\right| \leq \mathrm{C} r^{-1} \rho .
$$

\section{RANDOM PERTURBATIONS}

The density of a measure with respect to Lebesgue evolves as

$$
\mathcal{L} f:=f \circ T^{-1} \text {. }
$$

We will then construct a random perturbation by introducing the convolution operator

$$
Q_{\varepsilon} f(x):=\int_{\mathbb{T}^{2}} q_{\varepsilon}(x-y) f(y) d y .
$$

Where we assume

(1) $q_{\varepsilon}(\xi):=\varepsilon^{-2} \bar{q}\left(\varepsilon^{-1} \xi\right) ; \bar{q} \in \mathcal{C}^{\infty}\left(\mathbb{R}^{2}, \mathbb{R}^{+}\right) ;$

(2) $\int_{\mathbb{R}^{2}} \bar{q}(\xi) d \xi=1$

(3) $\bar{q}(\xi)=0$ for each $\|\xi\| \geq 1$;

(4) $\bar{q}(\xi)=1$ for each $\|\xi\| \leq \frac{1}{2}$.

We define then

$$
\mathcal{L}_{\varepsilon}:=Q_{\varepsilon} \mathcal{L}^{n_{\varepsilon}}
$$

where $n_{\varepsilon}$ will be chosen later.

Notice that

$$
\mathcal{L}_{\varepsilon}^{2} f(x)=\int_{\mathbb{T}^{4}} q_{\varepsilon}(x-y) q_{\varepsilon}\left(T^{-n_{\varepsilon}} y-T^{n_{\varepsilon}} z\right) f(z) d z d y:=\int_{\mathbb{T}^{2}} \mathcal{K}_{\varepsilon}(x, z) f(z) d z .
$$

We have thus a kernel operator that can be investigated with rather coarse techniques. It turns out to be convenient to define the associated kernel

$$
\overline{\mathcal{K}}_{\varepsilon}(x, z):=\mathcal{K}_{\varepsilon}\left(x, T^{-n_{\varepsilon}} z\right)=\int_{\mathbb{T}^{2}} q_{\varepsilon}(x-y) q_{\varepsilon}\left(T^{-n_{\varepsilon}} y-z\right) m(d y) .
$$

For further use let us define

$$
\begin{aligned}
& D_{r}:=\left\{z=\left(z_{1}, z_{2}\right) \in \mathbb{T}^{2}|| z_{1}|\leq r ;| z_{2} \mid \leq r^{2}\right\} \\
& B_{r}(\xi):=\left\{\eta \in \mathbb{T}^{2} ;\|\xi-\eta\|<r\right\} .
\end{aligned}
$$

The following is a relevant fact used extensively in the sequel.

Lemma 8.1. There exists $\mathrm{C}_{3}, R>0$ such that, for each $\delta<R^{2}$, if $B_{\delta}(\xi) \subset D_{R / 2}$, then there exists $\eta \in B_{\frac{3}{4} \delta}(\xi)$ such that $T^{n} B_{\frac{\delta}{4}}(\eta) \cap D_{R}=\emptyset$ for some $n \leq \mathrm{C}_{3} \delta^{-\frac{1}{2}}$.

Proof. If $\xi$ belongs to the first or third quadrant, then $T^{n} \xi$ is escaping from the origin. In such a case, if $B_{\frac{5}{8} \delta}(\xi)$ belongs to the thin sector we choose $\eta \in B_{\frac{\delta}{2}}(\xi),|y| \geq \frac{3}{8} \delta$. Clearly, if $(x, y) \in B_{\frac{\delta}{4}}(\eta)$, C $x^{2} \geq|y| \geq \frac{\delta}{4}$. On the other hand $\left|y_{n}\right| \geq n|x|^{3}$ while $\left|x_{n}\right| \leq|x|+n \mathrm{C}\left|x_{n}\right|^{2}$. So, if $n \leq \mathrm{C}|x|^{-1} \leq \mathrm{C} \delta^{-\frac{1}{2}}$, we have $\left|y_{n}\right| \geq \mathrm{C}\left|x_{n}\right|^{2}$. After that we can compare the dynamics with one of the type $x \mapsto x+\mathrm{C} x^{2}$, hence after a time at most $\mathrm{C} \delta^{-\frac{1}{2}}$ the $T^{n} B_{\frac{\delta}{4}}(\eta)$ will exit $D_{R}$. If the above does not apply, then one can take a ball of radius $\delta / 4$ belonging completely to the fat sector and centered at a point in $B_{\delta}(\xi)$. Then the results of subsection 4.1 easily implies the lemma. If, on the contrary, $\xi$ belong to the second or fourth quadrant, then its trajectory may approach the origin in an arbitrary manner (even asymptotically, if the point belongs to the stable manifold). In such a case we can take a point $\eta \in B_{\delta}(\xi)$ at, at least, a vertical distance $\frac{3}{4} \delta$ from the stable manifold and such that $B_{\frac{3}{4} \delta}(\eta) \subset B_{\delta}(\xi)$. Again from the results of subsection 4.1 it follows that such a ball will exit $D_{R}$ in a time at most $\mathrm{C}_{3} \delta^{-\frac{1}{2}}$.

Lemma 8.2. There exit constants $\sigma, \mathrm{C}_{3}>0$ such that if $n_{\varepsilon}=\mathrm{C}_{3} \varepsilon^{-\frac{1}{2}}$ holds

$$
\overline{\mathcal{K}}_{\varepsilon}(x, z) \geq \sigma \quad \forall x, z \in \mathbb{T}^{2} .
$$


Proof. It is trivial to see that

$$
\overline{\mathcal{K}}_{\varepsilon}(x, z) \geq \varepsilon^{-4} m\left(B_{\varepsilon / 2}(x) \cap T^{n_{\varepsilon}} B_{\varepsilon / 2}(z)\right) .
$$

Accordingly, by Lemma 8.1 there exists two balls, of radius $\frac{\varepsilon}{8}, \bar{B}_{1} \subset B_{\varepsilon / 2}(z)$ and $\bar{B}_{2} \subset B_{\varepsilon / 2}(x)$ that are outside of $D_{r}, r \geq \sqrt{\frac{\varepsilon}{2}}$, and whose images will be outside of a neighborhood of the origin or order one in a time less than $\mathrm{C} \varepsilon^{-\frac{1}{2}}$, forward and backward in time, respectively. Given two unstable manifolds in $B_{1}$ at a distance larger than $\bar{c} r \varepsilon$, for some appropriate $\bar{c}$, then no stable manifold will intersect both manifolds inside the ball $B_{1}$. We can thus consider $\mathrm{C} r^{-1}$ unstable manifolds such that no stable manifolds intersect two of them in $B_{1}$. Around each such manifold we can construct a strip by moving along the stable manifold by $\mathrm{C} \varepsilon$. We obtain in this way $\mathrm{C} r^{-1}$ disjoint strips each of area $\mathrm{C} r \varepsilon^{2}$, whose union covers a fixed fraction of the area of $B_{1}$. After a time less that $\mathrm{C} \varepsilon^{-\frac{1}{2}}$ such strips will be outside a neighborhood of zero, their length may have increase considerable, if so we will subdivide them into strips of length $\varepsilon$. Since now the stable and unstable manifold are at a fixed angle and by the usual distortion arguments, such strips are essentially rectangular. At this point, by Lemma [5.1] it will suffice to wait a time $\varepsilon^{-\frac{1}{2}}$ to insure that each such strip will acquire length at least $\frac{1}{2}$ in the unstable direction. We thus iterate for such a time and, if one strip becomes longer than one, we subdivide it into pieces of length between $\frac{1}{2}$ and one. Finally, fix some box $\Lambda$ of some fixed size $C$ away from the origin with sides approximately parallel either to the stable or to the unstable directions. By mixing it suffices to wait a fixed time to be sure that a fixed percentage of each one of the above mentioned strips will intersect the box. In addition, it is possible to insure that such strips cut the box from one stable side to the other.

We can then write $m\left(B_{\varepsilon / 2}(x) \cap T^{n_{\varepsilon}} B_{\varepsilon / 2}(z)\right)=m\left(T^{-n_{\varepsilon} / 2} B_{\varepsilon / 2}(x) \cap T^{n_{\varepsilon} / 2} B_{\varepsilon / 2}(z)\right)$ since the same considerations done above for the unstable manifold can be done, iterating backward, for the stable manifold it follows that a fixed percentage of $T^{-n_{\varepsilon} / 2} B_{\varepsilon / 2}(x)$ and a fixed percentage of $\left.T^{n_{\varepsilon} / 2} B_{\varepsilon / 2}(z)\right)$ will intersect $\Lambda$ and hence each other. In fact each one of the above constructed strips in the unstable direction will intersects each one of the strips in the stable direction. By the usual distortion estimates, this implies that the intersection among any two such strip has a measure proportional to the product of the measure of the two strips, hence

and the lemma.

$$
m\left(B_{\varepsilon / 2}(x) \cap T^{n_{\varepsilon}} B_{\varepsilon / 2}(z)\right) \geq \mathrm{C} m\left(B_{\varepsilon / 2}(x)\right) m\left(B_{\varepsilon / 2}(z)\right),
$$

Lemma 8.3. For each $f \in L^{1}, \int f=0$ holds

$$
\left\|\mathcal{L}_{\varepsilon}^{n} f\right\|_{1} \leq(1-\sigma)^{n / 2}\|f\|_{1} .
$$

Proof. Note that $\mathcal{L}_{\varepsilon} 1=\mathcal{L}_{\varepsilon}^{*} 1=1$ and let $\mathcal{M}_{\varepsilon}^{+}=\left\{x \in \mathbb{T}^{2} \mid \mathcal{L}_{\varepsilon}^{2} f \geq 0\right\} ; \mathcal{M}_{+}=\left\{x \in \mathbb{T}^{2} \mid f \geq 0\right\}$, then, since $\int \mathcal{L}_{\varepsilon} f=\int f=0$

$$
\begin{aligned}
\left\|\mathcal{L}_{\varepsilon}^{2} f\right\|_{1} & =2 \int_{\mathcal{M}_{\varepsilon}^{+}} d x \mathcal{L}_{\varepsilon}^{2} f=2 \int_{\mathcal{M}_{\varepsilon}^{+}} d x \int_{\mathbb{T}^{2}} d y \mathcal{K}_{\varepsilon}(x, y) f(y) \\
& =2 \int_{\mathbb{T}^{2}} d y f(y) \int_{\mathcal{M}_{\varepsilon}^{+}} d x\left[\mathcal{K}_{\varepsilon}(x, y)-\sigma\right] \\
& \leq 2 \int_{\mathcal{M}_{+}} d y f(y) \int_{\mathbb{T}^{2}} d x\left[\mathcal{K}_{\varepsilon}(x, y)-\sigma\right]=2(1-\sigma) \int_{\mathcal{M}_{+}} d y f(y) \\
& =(1-\sigma)\|f\|_{1} .
\end{aligned}
$$

Let $v^{u, s}=\left(v_{1}^{u, s}, v_{2}^{u, s}\right)$ be the unit tangent vector fields in the unstable and stable direction, respectively. Clearly $\left|\partial^{u}\left(\mathcal{L}^{i} f\right)\right| \leq\left|\partial^{u} f\right|$, while $\left|\partial^{s}\left(g \circ T^{i}\right) \leq\right| \partial^{s} g \mid$.

Lemma 8.4. For each $f, g \in \mathcal{C}^{1}\left(\mathbb{T}^{2}, \mathbb{R}\right)$ holds

$$
\left|\int Q_{\varepsilon} f g-\int f g\right| \leq \mathrm{C} \varepsilon\left\{\|f\|_{\infty}+\left\|\partial^{u} f\right\|_{L^{1}(\nu)}\right\}\left\{\|g\|_{\infty}+\left\|\partial^{s} g\right\|_{L^{1}(\nu)}\right\}
$$

where $\nu$ is the measure defined by $\nu(h):=\int d \rho \int_{\partial D_{\rho}} h$. 
Proof. It is convenient to introduce the following change of variables. For each $x, y \in \mathbb{T}^{2}$ close enough, let us call $[x, y]=W_{\delta}^{u}(x) \cap W_{\delta}^{s}(y)$, note that by Lemma 4.1 such a point is always well defined provided $d(x, y) \leq \mathrm{C} \delta^{2}$. We consider then the change of variable $\Phi: \mathbb{T}^{2} \times \mathbb{T}^{2} \rightarrow \mathbb{R}^{2} \times \mathbb{T}^{2}$,

$$
\begin{aligned}
& \xi:=x-y \\
& \eta:=[x, y] .
\end{aligned}
$$

Due to the absolute continuity of the holonomies the above change of variable is absolutely continuous. Clearly, $\partial_{x}^{u} \eta=0$ since moving along $W^{u}(x)$ does not change the intersection point with $W^{s}(y)$. On the other hand $\partial_{x}^{s} \eta=J \Psi_{x}^{u} v^{s}(\eta)$, since moving $x$ along $W^{s}(x)$ moves $\eta$ on $W^{s}(y)$ by an amount determined exactly by the unstable holonomy $\Psi^{u}$ between $W^{s}(x)$ and $W^{s}(y)$. By similar arguments and a straightforward computations

$$
\begin{aligned}
& \partial_{x_{1}} \eta=v_{2}^{u}(x) \operatorname{det}\left(v^{s}(x) \quad v^{u}(x)\right)^{-1} J \Psi_{x}^{u} v^{s}(\eta) \\
& \partial_{x_{2}} \eta=v_{1}^{u}(x) \operatorname{det}\left(v^{u}(x) \quad v^{s}(x)\right)^{-1} J \Psi_{x}^{u} v^{s}(\eta) \\
& \partial_{y_{1}} \eta=v_{2}^{s}(y) \operatorname{det}\left(v^{u}(y) \quad v^{s}(y)\right)^{-1} J \Psi_{y}^{s} v^{u}(\eta) \\
& \partial_{y_{2}} \eta=v_{1}^{s}(y) \operatorname{det}\left(v^{s}(y) \quad v^{u}(y)\right)^{-1} J \Psi_{y}^{s} v^{u}(\eta) .
\end{aligned}
$$

In fact, calling $\theta(x)$ the sine of the angle between stable and unstable directions at the point $x$ and $v_{\perp}$ the orthogonal unit vector to $v$, holds

$$
\begin{aligned}
& \partial_{x} \eta=J \Psi_{x}^{u} \theta(x)^{-1}\left|v^{s}(y)\right\rangle\left\langle v_{\perp}^{u}(x)\right| \\
& \partial_{y} \eta=J \Psi_{y}^{s} \theta(y)^{-1}\left|v^{u}(x)\right\rangle\left\langle v_{\perp}^{s}(y)\right| .
\end{aligned}
$$

Accordingly,

$$
\begin{aligned}
J \Phi & :=\operatorname{det}\left(\begin{array}{cc}
\mathbf{I d} & -\mathbf{I d} \\
\partial_{x} \eta & \partial_{y} \eta
\end{array}\right)=\operatorname{det}\left(\partial_{x} \eta+\partial_{y} \eta\right) \\
& =J \Psi_{x}^{u} J \Psi_{y}^{s} \theta(x)^{-1} \theta(y)^{-1}\left\langle v_{\perp}^{s}(y), v^{u}(x)\right\rangle^{2} \\
& =J \Psi_{x}^{u} J \Psi_{y}^{s} \theta(x)^{-1} \theta(y)^{-1} \operatorname{det}\left(v^{u}(x) \quad v^{s}(y)\right)^{2} .
\end{aligned}
$$

Before starting computing we need to collect some facts.

Sub-lemma 8.5. If $x \in \partial D_{2 \rho}, \rho \geq r \geq \mathrm{C}_{4} \sqrt{\varepsilon}$, then, for $C_{4}$ large enough,

i) if $\|x-y\| \leq \varepsilon$, then $y \notin D_{\rho}$.

ii) $\|x-\eta\| \leq \frac{\varepsilon}{\rho}$.

iii) if $\zeta \in W^{u}(x)$ and $\|\zeta-x\| \leq \frac{\varepsilon}{\rho}$, then $\zeta \notin D_{\rho}$.

Proof. The first inequality follows since $D_{2 \rho}$ has a vertical size $4 \rho^{2}$. Thus $2 \rho^{2}-\varepsilon \geq 2 \rho^{2}-\frac{1}{\mathrm{C}_{4}^{2}} \rho^{2} \geq \rho^{2}$. Such an estimate and Proposition 4.1 imply that the angle between $W^{u}(x)$ and $W^{u}(y)$ is at least $4 K_{-} \rho^{-1}$, thus (ii). Finally, Proposition 4.1 implies that, if $\zeta=\left(z_{1}, z_{2}\right), z_{2}>2 \rho^{2}-\frac{\varepsilon^{2}}{\rho^{2}} \geq \rho^{2}$.

We can now start computing the integral.

$$
\begin{aligned}
\int_{\mathbb{T}^{4}} d x d y f(x) g(y) q_{\varepsilon}(x-y) & =\int_{D_{\mathrm{C}_{4} r}^{c}} d x \int_{\mathbb{T}^{2}} d y f(x) g(y) q_{\varepsilon}(x-y)+\mathcal{O}\left(\|f\|_{\infty}\|g\|_{\infty} r^{3}\right) \\
& \geq \int_{\Phi\left(D_{\mathrm{C}_{4} r}^{c} \times \mathbb{T}^{2}\right)} d \eta d \xi f(x) g(y) q_{\varepsilon}(\xi) J \Phi^{-1}+\|f\|_{\infty}\|g\|_{\infty} \mathcal{O}\left(r^{3}\right)
\end{aligned}
$$

Next, from formula [8.7) and Sub-lemma 8.5. (i) follows

$$
|J \Phi(x, y)-1| \leq \mathrm{C} \frac{\varepsilon}{\rho^{2}} .
$$


Hence,

$$
\begin{aligned}
\int_{\mathbb{T}^{4}} d x d y f(x) g(y) q_{\varepsilon}(x-y)= & \int_{\mathbb{T}^{2}} d \eta f(\eta) g(\eta)+\|f\|_{\infty}\|g\|_{\infty} \mathcal{O}\left(\int_{D_{r}^{c}}\left|1-J \Phi^{-1}\right|\right) \\
& +\mathcal{O}\left(\int_{\Phi\left(D_{\mathrm{C}_{4} r}^{c} \times \mathbb{T}^{2}\right)} d \eta d \xi[f(x)-f(\eta)] g(y) q_{\varepsilon}(\xi)\right) \\
& +\mathcal{O}\left(\int_{\Phi\left(D_{\mathrm{C}_{4} r}^{c} \times \mathbb{T}^{2}\right)} d \eta d \xi f(\eta)[g(y)-g(\eta)] q_{\varepsilon}(\xi)\right) \\
& +\|f\|_{\infty}\|g\|_{\infty} \mathcal{O}\left(r^{3}\right) .
\end{aligned}
$$

To conclude we must compute the various error terms. For each $f \in L^{\infty}$, holds

$$
\int_{D_{R}} d x f(x)=\frac{2}{3} \int_{0}^{R} d \rho \frac{\rho}{1+\rho} \int_{\partial D_{\rho}} d s f(s) .
$$

Remembering (8.8) and applying Fubini

$$
\int_{D_{r}^{c}}\left|1-J \Phi^{-1}\right| \leq \mathrm{C}\|f\|_{\infty} \int_{r}^{1} d \rho \rho^{2} \frac{\varepsilon}{\rho^{2}} \leq \mathrm{C}\|f\|_{\infty} \varepsilon .
$$

Next, let $\gamma_{u}^{\eta}:[-\delta, \delta] \rightarrow \mathbb{T}^{2}$ be the unstable manifold of $\eta$, parametrized by arc-length, and let $s(\eta, \xi)$ be such that $\gamma_{u}^{\eta}(s(\eta, \xi))=x$. Recalling Sub-lemma 8.5

$$
\begin{aligned}
\left|\int_{\Phi\left(D_{\mathrm{C}_{4} r}^{c} \times \mathbb{T}^{2}\right)} d \eta d \xi[f(x)-f(\eta)] g(y) q_{\varepsilon}(\xi)\right| & \leq\|g\|_{\infty} \int_{\Phi\left(D_{\mathrm{C}_{4} r}^{c} \times \mathbb{T}^{2}\right)} d \eta \int_{0}^{s(\eta, \xi)} d t\left|\partial^{u} f\left(\gamma_{u}^{\eta}(t)\right)\right| q_{\varepsilon}(\xi) \\
& \leq \mathrm{C}\|g\|_{\infty} \int_{D_{r}^{c} \times \mathbb{T}^{2}} d \eta^{\prime} d \xi\left|\partial^{u} f\left(\eta^{\prime}\right)\right| \frac{\|\xi\|}{\left|\theta\left(\eta^{\prime}\right)\right|} q_{\varepsilon}(\xi) \\
& \leq \mathrm{C}\|g\|_{\infty} \int_{0}^{1} d \rho \varepsilon \int_{\partial D_{\rho}}\left|\partial^{u} f\right| \\
& \leq \mathrm{C}\|g\|_{\infty}\left\|\partial^{u} f\right\|_{L^{1}(\nu)} \varepsilon
\end{aligned}
$$

Analogously,

$$
\left|\int_{D_{r}^{c} \times \mathbb{T}^{2}} d \eta d \xi f(\eta)[g(y)-g(\eta)] q_{\varepsilon}(\xi)\right| \leq \mathrm{C}\|f\|_{\infty}\left\|\partial^{s} g\right\|_{L^{1}(\nu)} \varepsilon .
$$

We can finally collect all the above estimates and obtain

$$
\begin{aligned}
\int_{\mathbb{T}^{4}} d x d y & f(x) g(y) q_{\varepsilon}(x-y)=\int_{\mathbb{T}^{2}} d \eta f(\eta) g(\eta) \\
& +\left(\|f\|_{\infty}+\left\|\partial^{u} f\right\|_{L^{1}(\nu)}\right)\left(\|g\|_{\infty}+\left\|\partial^{s} g\right\|_{L^{1}(\nu)}\right) \mathcal{O}\left(r^{3}+\varepsilon\right)
\end{aligned}
$$

from which the lemma follows by choosing $r=\varepsilon^{\frac{1}{3}}$.

\section{DECAY OF CORRELATIONS}

Here we put together the results of the previous section to prove Theorem 2.4

Let $f, g \in \mathcal{C}^{1}\left(\mathbb{T}^{2}, \mathbb{R}\right), \int f=0$, then

$$
\begin{aligned}
\int \mathcal{L}^{k n_{\varepsilon}} f g & =\sum_{i=0}^{k-1} \int \mathcal{L}^{n_{\varepsilon} i}\left(\mathcal{L}^{n_{\varepsilon}}-\mathcal{L}_{\varepsilon}\right) \mathcal{L}_{\varepsilon}^{k-i-1} f g+\int \mathcal{L}_{\varepsilon}^{k} f g \\
& =\sum_{i=0}^{k-1} \int\left(\mathcal{L}^{n_{\varepsilon}}-\mathcal{L}_{\varepsilon}\right) \mathcal{L}_{\varepsilon}^{k-i-1} f g \circ T^{n_{\varepsilon} i}+\mathcal{O}\left(e^{-\mathrm{C} k}\|f\|_{1}\|g\|_{\infty}\right) \\
& =\sum_{i=0}^{k-1} \int\left(\mathbf{I} \mathbf{d}-Q_{\varepsilon}\right) \mathcal{L}^{n_{\varepsilon}} \mathcal{L}_{\varepsilon}^{k-i-1} f g \circ T^{n_{\varepsilon} i}+\mathcal{O}\left(e^{-\mathrm{C} k}\|f\|_{1}\|g\|_{\infty}\right),
\end{aligned}
$$


where we have used Lemma 8.3 To conclude, by using Lemma 8.4 we need to estimate the $L^{1}$ norm of $\partial^{u}\left(\mathcal{L}^{n_{\varepsilon}} \mathcal{L}_{\varepsilon}^{j} f\right)$. Since $\left|D \mathcal{L}_{\varepsilon}^{j} f\right| \leq \mathrm{C} \varepsilon^{-1}|f|_{\infty}$, for $j>0$,

$$
\begin{aligned}
\left\|\partial^{u}\left(\mathcal{L}^{n_{\varepsilon}} \mathcal{L}_{\varepsilon}^{j} f\right)\right\|_{L^{1}(\nu)} & \leq \int_{0}^{\mathrm{C}_{4} \sqrt{\varepsilon}} d \rho \int_{\partial D_{\rho}}\left|\partial^{u}\left(\mathcal{L}^{n_{\varepsilon}} \mathcal{L}_{\varepsilon}^{j} f\right)\right|+\int_{\mathrm{C}_{4} \sqrt{\varepsilon}}^{1} d \rho \int_{\partial D_{\rho}}\left|\partial^{u}\left(\mathcal{L}^{n_{\varepsilon}} \mathcal{L}_{\varepsilon}^{j} f\right)\right| \\
& \leq \mathrm{C}\|f\|_{\infty} \int_{0}^{\mathrm{C}_{4} \sqrt{\varepsilon}} d \rho \varepsilon^{-1} \rho+\mathrm{C}|f|_{\infty} \int_{\mathrm{C}_{4} \sqrt{\varepsilon}}^{1} d \rho \int_{\partial D_{\rho}} \frac{\varepsilon}{\rho^{2}} \varepsilon^{-1} \\
& \leq \mathrm{C}\|f\|_{\infty} \ln \varepsilon^{-1}
\end{aligned}
$$

where we have used Lemma 5.1 and Sub-lemma $8.5{ }^{14}$

Thus

$$
\left|\int \mathcal{L}^{n} f g\right|=\left(\|f\|_{\infty}+\left\|\partial^{u} f\right\|_{L^{1}(\nu)}\right)\|g\|_{\mathcal{C}^{1}} \mathcal{O}\left(n \varepsilon^{\frac{3}{2}} \ln \varepsilon^{-1}+e^{-\mathrm{C} n \sqrt{\varepsilon}}\right) .
$$

Clearly the best choice is $\varepsilon=\mathrm{C}\left(n^{-1} \ln n\right)^{2}$ which implies the Theorem.

\section{LOWER BOUND}

In this section we prove a lower bound.

Lemma 10.1. If there exists a sequence $\gamma_{n}$ such that, for each $f, g \in \mathcal{C}^{1}$ holds

$$
\left|\int_{\mathbb{T}^{2}} f \circ T^{-n} g-\int_{\mathbb{T}^{2}} f \int_{\mathbb{T}^{2}} g\right| \leq\left(\left\|\partial^{u} f\right\|_{L^{1}(\nu)}+\|f\|_{L^{1}(\nu)}\right)|g|_{\mathcal{C}^{1}} \gamma_{n}
$$

then there exists $\mathrm{C}>0$ such that

$$
\gamma_{n} \geq n^{-2} \mathrm{C} .
$$

Proof. Let $g \geq 0$ be a smooth function supported away from zero (let us say that the support of $g$ does not intersect $\left.D_{\frac{1}{2}}\right)$. Next, let $\xi_{0}=\left(x_{0}, y_{0}\right)=\left(\frac{1}{2}, y_{0}\right) \in W^{u}(0), \xi_{n}=T^{-n} \xi_{0}$. For each point $\eta$ in a neighborhood of $\xi_{n}$ let $z_{1}$ be the distance, along $W^{u}(0)$, between $\xi_{n}$ and $W^{u}(0) \cap W^{s}(\eta)$, and $z_{2}$ the distance, along $W^{s}\left(\xi_{n}\right)$, between $\xi_{n}$ and $W^{u}\left(\xi_{n}\right) \cap W^{s}(\eta)$. By construction $\Xi_{n}(\eta):=\left(z_{1}, z_{2}\right)$ is a map from a neighborhood of $\xi_{n}$ to a neighborhood of the origin with the property that the map transforms the stable and unstable foliation into the standard foliation given by the Cartesian coordinates. Clearly, $\Xi_{n}^{-1}(s, 0)=\gamma^{u}(s)$ (the unstable manifold of the origin parametrized by arc length and such that $\left.\gamma^{u}(0)=\xi_{n}\right)$, while $\Xi_{n}^{-1}(0, s)=\gamma_{n}^{s}(s)$ (the unstable manifold of $\xi_{n}$ parametrized by arc length). Finally we define $f_{n}:=\left(\alpha_{n} \beta_{n}\right) \circ \Xi_{n}$ with $\alpha_{n}\left(z_{1}\right):=\varsigma\left(\mathrm{C}_{5} n z_{1}\right)$, $\beta_{n}\left(z_{2}\right):=\varsigma\left(\mathrm{C}_{5} n^{-1} z_{2}\right)$, for $C_{5}$ small enough, and

$$
\varsigma(x):= \begin{cases}1-|x+1| & |x+1| \leq 1 \\ 0 & |x+1|>1 .\end{cases}
$$

In other words, $f_{n}$ is a function essentially supported on a neighborhood left of $\xi_{n}$ of order $n^{-1}$ in the unstable direction and the stable. Accordingly, the supports of $f_{n} \circ T^{-k}$ and $g$ are disjoint for all $k \leq \mathrm{C} n$. Lemma 7.1 implies that the the support is essentially a rhombus of size $n^{-1}$ and angle $n^{-1}$.

Thus we have

$$
|g|_{\mathcal{C}^{1}}\left(\left\|\partial^{u} f_{n}\right\|_{L^{1}(\nu)}+\left\|f_{n}\right\|_{L^{1}(\nu)}\right) \gamma_{n} \geq \int_{\mathbb{T}^{2}} f_{n} \int_{\mathbb{T}^{2}} g \geq \mathrm{C} n^{-3}
$$

On the other hand, using again Lemma 7.1

$$
\left\|\partial^{u} f_{n}\right\|_{L^{1}(\nu)} \leq \mathrm{C} n\left\|\left(\alpha^{\prime} \beta\right) \circ \Xi_{n}\right\|_{L^{1}(\nu)} \leq \mathrm{C} n^{-1}
$$

which yields the Lemma.

\footnotetext{
${ }^{14}$ The above estimate is not sharp. With some extra work one could avoid the $\ln \varepsilon^{-1}$, yet this would not change in any substantial way the result, so we chose to keep the presentation as short as possible.
} 
Remark 10.2. Note that the norms in Lemma 10.1 and in Theorem 2.4 (even in the stronger version given by (9.1) ) are different. It is not obvious that, putting the $L^{\infty}$ norm instead of the $L^{1}(\nu)$ one keeps the same rate of mixing. More generally, it is well known that in the uniformly hyperbolic setting the smoothness of the function can have an influence on the mixing rate. An analogous effect may arise in the present setting but it remains to be investigate. A related problem that needs to be addressed is the higher dimensional analogous of the present model where the fixed point has different possibility of losing full hyperbolicty. It is clear that the present result is only the starting point and not the end of the story.

\section{REFERENCES}

[1] Artuso, R., Prampolini, A., Correlation decay for an intermittent area-preserving map, Physics Letters A, 246 (1998) 407-411.

[2] G.Benettin, A.Giorgilli, On the Hamiltonian interpolation of near-to-identity symplectic mappings with application to symplectic integration algorithms, Journal of Statistical Physics, 74, n. 5/6, (1994), 1117-1143.

[3] Bleher, P. M., Statistical properties of two-dimensional periodic Lorentz gas with infinite horizon, J. Statist. Phys. 66 (1992), no. 1-2, 315-373.

[4] Bunimovich, L. A.; Sinai, Ya. G., Statistical properties of Lorentz gas with periodic configuration of scatterers, Comm. Math. Phys. 78 (1980/81), no. 4, 479-497.

[5] Gouëzel, S., Sharp polynomial estimates for the decay of correlations, Israel J. Math. 139 (2004), 29-65.

[6] Gouëzel, S., Central limit theorem and stable laws for intermittent maps, Probab. Theory Related Fields 128 (2004), no. $1,82-122$.

[7] A.Katok, B.Hasselblatt, Introduction to the Modern Theorey of Dynamical Systems, Encyclpedia of Mathematical and its Applicatios, 54, Cambridge University press, Cambridge (1995).

[8] Hu, H., Decay of correlations for piecewise smooth maps with indifferent fixed points, Ergodic Theory Dynam. Systems 24 (2004), no. 2, 495-524.

[9] Hu, H., Statistical properties of some almost hyperbolic systems Smooth ergodic theory and its applications (Seattle, WA, 1999), 367-384, Proc. Sympos. Pure Math., 69, Amer. Math. Soc., Providence, RI, 2001.

[10] Liverani C., Flows, Random Perturbations and Rate of Mixing, Ergodic Theory and Dynamical Systems, 18, 6, pp. 14211446 (1998).

[11] Liverani C.,Birth of an elliptic island in a chaotic sea, Mathematical Physics Electronic Journal, 10, 1 (2004).e

[12] Liverani C., Central Limit Theorem for Deterministic Systems, International Conference on Dynamical Systems, Montevideo 1995, a tribute to Ricardo Mañe, Pitman Research Notes in Mathemaics Series, 362, editors F.Ledrappier, J.Levovicz, S.Newhouse, (1996).

[13] Liverani C.,, Saussol B., Vaienti S., A Probabilistic Approach to Intermittency, Ergodic Theory and Dynamical Systems, 19, pp. 671-685 (1999).

[14] Liverani C., Wojtkowski M., Ergodicity in Hamiltonian Systems, in Dynamics Reported 4, Springer, Berlin, Heidelbeg, (1995).

[15] Maume, V. Projective metrics and mixing properties on towers, Trans. Am. Math. Soc. 353, 3371-3389 (2001).

[16] Pollicott, M., Rates of mixing for potentials of summable variation Trans. Am. Math. Soc. 352, 843-853 (2000).

[17] Pollicott, M., Sharp, R. Invariance principles for interval maps with an indifferent fixed point, Comm. Math. Phys. 229 (2002), no. 2, 337-346.

[18] Pollicott, M., Yuri, M. Statistical properties of maps with indifferent periodic points, Commun. Math. Phys. 217, 503-520 (2001).

[19] Y. Pomeau, P. Manneville, Intermittent transition to turbulence in dissipative dynamical systems, Communication in Mathematical Physics, 74, 189-197 (1980).

[20] Sarig, O.M., Thermodynamic Formalism for Countable Markov Shifts, Ergodic Theory Dyn. Syst. 19, 1565-1593 (1999)

[21] Wang X.J., Statistical physics of temporal intermittency, Phys. Rev. A 40,6647 (1989).

[22] Young L.-S., Recurrence times and rates of mixing. Israel J. Math. 110 (1999), 153-188.

[23] Yuri M., Decay of correlations for certain multi-dimensional maps, Nonlinearity, 9, n. 6 (1996) 1439-1461.

[24] Zaslavsky, G. M., Chaos, fractional kinetics, and anomalous transport, Phys. Rep. 371 (2002), no. 6, 461-580.

[25] Zweimüller, R., Stable limits for probability preserving maps with indifferent fixed points, Stoch. Dyn. 3 (2003), no. 1, 83-99.

Carlangelo Liverani, Dipartimento di Matematica, it Università di Roma (Tor Vergata), Via della Ricerca SCIENTIFica, 00133 Roma, Italy.

E-mail address: liverani@mat.uniroma2.it

Marco Martens, University of Groningen, Department of Mathematics P.O. Box 800, 9700 AV Groningen, The NETHERLANDS

E-mail address: m.martens@math.rug.nl 